\title{
Modification of the In Vitro Uptake Mechanism and Anti-Oxidant Levels in HaCaT Cells and Resultant Changes to Toxicity and Oxidative Stress of G4 and G6 Poly (Amido Amine) Dendrimer
}

\section{Nanoparticles}

\author{
Marcus A. Maher \\ Technological University Dublin \\ Hugh Byrne \\ Technological University Dublin, hugh.byrne@tudublin.ie
}

Follow this and additional works at: https://arrow.tudublin.ie/biophonart

Part of the Biological and Chemical Physics Commons

\section{Recommended Citation \\ "Modification of the in vitro uptake mechanism and anti-oxidant levels in HaCaT cells and resultant changes to toxicity and oxidative stress of G4 and G6 Poly (amido amine) dendrimer nanoparticles", Marcus A. Maher and Hugh J. Byrne, Analytical and Bioanalytical Chemistry, 408, 5295-5307 (2016)}

This Article is brought to you for free and open access by the DIT Biophotonics and Imaging at ARROW@TU Dublin. It has been accepted for inclusion in Articles by an authorized administrator of ARROW@TU Dublin. For more information, please contact arrow.admin@tudublin.ie, aisling.coyne@tudublin.ie,gerard.connolly@tudublin.ie. Funder: DIT

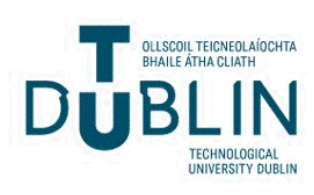


1 Modification of the in vitro uptake mechanism and anti-oxidant levels in

2 HaCaT cells and resultant changes to toxicity and oxidative stress of G4

3 and G6 Poly (amido amine) dendrimer nanoparticles.

5 Marcus A. Maher ${ }^{1,2, *}$, Hugh J. Byrne ${ }^{1}$

6

$7 \quad{ }^{1}$ FOCAS Research Institute, Dublin Institute of Technology, Kevin Street, Dublin 8, Ireland

$8 \quad{ }^{2}$ School of Physics, Dublin Institute of Technology, Kevin Street, Dublin 8, Ireland

9

$10 *$ Corresponding author: marcus.maher@mydit.ie

11

12

13 Keywords:

14 Dendrimer nanoparticles

15 PAMAM nanoparticles

16 Endocytosis

17 Passive diffusion

18 Oxidative stress

19 Membrane permeability 


\section{Abstract:}

21 The mechanism of cellular uptake by endocytosis and subsequent oxidative stress has been

22 identified as the paradigm for the toxic response of cationically surface charged nanoparticles. In

23 an attempt to circumvent the process, the effect of increased cellular membrane permeability on

24 the uptake mechanisms of poly (amidoamine) dendrimers generation 4 (G4) and 6 (G6) in vitro

25 was investigated. Immortalised, non-cancerous human keratinocyte (HaCaT) cells were treated

26 with DL-Buthionine-(S,R)-sulfoximine (BSO). Active uptake of the particles was monitored

27 using fluorescence microscopy to identify and quantify endosomal activity and resultant

28 oxidative stress, manifest as increased levels of reactive oxygen species, monitored using the

29 carboxy- $\mathrm{H}_{2}$ DCFDA dye. Dose dependent cytotoxicity for G4 and G6 exposure was registered

30 using the cytotoxicity assays Alamar Blue and MTT, from 6 to 72 hours.

31 Reduced uptake by endocytosis is observed for both dendrimer species. A dramatic change,

32 compared to untreated cells, is observed in the cytotoxic and oxidative stress response of the

33 BSO treated cells. The significantly increased mitochondrial activity, dose dependent anti-

34 oxidant behaviour and reduced degree of endocytosis for both dendrimer generations, in BSO

35 treated cells, indicates enhanced permeability of the cell membrane, resulting in the passive,

36 diffusive uptake of dendrimers, replacing endocytosis as the primary uptake mechanism. The

37 complex MTT response reflects the importance of glutathione in maintaining redox balance

38 within the mitochondria. The study highlights the importance of regulation of this redox balance

39 for cell metabolism, but also points to the potential of controlling the nanoparticle uptake

40 mechanisms, and resultant cytotoxicity, with implications for nanomedicine. 


\section{Introduction}

43 Nanoparticle science is a rapidly advancing field which holds much promise in areas such as

44 targeted drug and gene delivery. ${ }^{1,2,3}$ However, nanoparticle uptake into a cell has been

45 demonstrated, particularly for nanoparticles with an effective cationic surface charge, to give rise

46 to cytotoxic responses, raising concerns about the potential health and environmental impact of

47 the proliferation of nanomaterials in consumer products. ${ }^{4}$ A systematic understanding of the

48 mechanisms of toxicity and their dependence on nanoparticle physico-chemical properties on a

49 cellular level is therefore required. ${ }^{5}$ In the context of nanomedical applications, understanding

50 and controlling the uptake process and subcellular trafficking of the delivery vehicle and the

51 bioavailability of the cargo are critically important.

53 Cellular uptake of nanoparticles principally occurs via endocytosis, whereby the nanoparticle is

54 invaginated by the cellular membrane and is transported into the cell. ${ }^{6}$ As the low $\mathrm{pH}$

55 environment of the endosome attempts to digest the nanoparticle, the redox balance of the cell is

56 disrupted, and, in the case of nanoparticles with an effective cationic surface charge, the process

57 gives rise to an increase in the production of Reactive Oxygen Species (ROS), localised mainly

58 around the endosome (or later lysosome). ${ }^{6,7,9}$ Although intra cellular anti-oxidants attempt to

59 neutralise the imbalance, ROS production can be sufficient to lead to oxidative stress. ${ }^{10}$

60 Subsequently, a cascade of events and the release of several characteristic cytokines and

61 chemokines occurs, ultimately leading to cell death, a process which is the accepted paradigm of

62 nanotoxicity of many nanoparticles in vitro. ${ }^{6}$ The process has been well demonstrated for model

63 nanoparticle system such as amine functionalised polystyrene, ${ }^{11,12,13}$ amorphous nanosilica, ${ }^{14}$ and

64 nanomeric polymeric dendrimers. ${ }^{10}$ 
66 Aminated molecules are intrinsic antioxidants, however, and generally regarded as ROS

67 scavengers. As examples, spermine and spermidine have both been shown to reduce $\mathrm{Fe}^{3+}$ to $\mathrm{Fe}^{2+}$

$68 \quad 15$ and the ferric reducing activity of these molecules has been identified as a measure of anti-

69 oxidant potential. ${ }^{16}$ Carnosine, an endogenous dipeptide, has been shown to scavenge both

70 reactive oxygen and nitrogen species. ${ }^{17}$ Notably, in a study by Khalid et al. (2015), while the

71 larger, higher generations of the aminated nanoscale dendrimers Poly (propylene imine) (PPI)

72 were demonstrated to elicit oxidative stress and significant toxicity, the smaller, lower

73 generations exhibited intracellular antioxidant behaviour and low toxicity. ${ }^{18}$ Examination of the

74 uptake mechanisms indicated a transition from cellular uptake by passive diffusion at low

75 generations to active endocytosis for higher generations. The study suggests that, in the case of

76 catatonically charge nanoparticles, the endocytotic uptake and trafficking process is, in itself, a

77 source of cellular toxicity. In the context of drug delivery, invagination of the delivery vehicle

78 and cargo in this harsh environment may also be undesirable, and although escape by

79 endosomolysis is a potential strategy, ${ }^{19}$ this too can be a harsh process, causing significant

80 damage to the cell. ${ }^{20,21}$ For these reasons, circumventing endocytosis appears to be a valid

81 strategy for the reduction of toxicity associated with aminated nanoparticles as well as

82 nanoparticle drug or gene delivery.

84 Guarnieri et al. (2015), demonstrated that functionalization of aminated polystyrene

85 nanoparticles with the viral peptide gH625 (derived from Herpes simplex virus - 1), which has a

86 membrane perturbing domain, enables translocation of particles to the cytoplasm, avoiding

87 endocytosis and thus, dramatically reducing the cytotoxicity. ${ }^{22}$ An alternative strategy to increase 
88 the permeability of the cellular membrane, in vitro, is through the application of DL-Buthionine-

$89(S, R)$-sulfoximine (BSO). BSO has previously been employed to study the effects of the

90 reduction of levels of the intracellular antioxidant glutathione (GSH); and therefore oxidative

91 stress $^{23,24,25,26}$ and has been shown to cause membrane permeabilisation. ${ }^{27,28}$ BSO works as an

92 inhibitor of the enzyme Glutamate Cysteine Ligase (EC 6.3.2.2) (historically known as gamma-

93 glutamylcysteine synthetase) which is the first step in the production of GSH in the cell. ${ }^{29}$ This

94 reduction in GSH causes several different effects, one being the induction of the membrane

95 permeability transition in the mitochondria. ${ }^{30,31,32}$ The opening of this pore and the depletion of

96 GSH allows ROS to diffuse from the mitochondria to the cell and via lipid peroxidation, cause

97 damage to the cell membrane, ${ }^{28}$ leading to increased permeability.

99 The reduction of GSH in the cell can have other adverse effects, however, mainly due to the fact

100 that GSH is one of the main antioxidants involved in maintaining the redox balance of the

101 mitochondria. ${ }^{33}$ In the mitochondria, endogenous ROS is produced as a by-product of normal

102 metabolism and therefore completely eliminating GSH can leave the cell susceptible to damage

103 from this ROS. $^{34}$ Furthermore, reductions in cellular GSH can also lead to changes in the

104 regulation of $\mathrm{Ca}^{2+}$ distribution ${ }^{35,36,37}$ and the activation/deactivation of signalling pathways

105 involved with growth, differentiation and apoptosis. ${ }^{33,38,39,40}$ The loss of GSH and subsequent

106 changes in the cell have been implicated in several disease states which is reviewed elsewhere. ${ }^{34}$

108 This study explores the impact, for HaCaT cell, of BSO treatment on the cellular uptake of, and

109 subsequent oxidative stress and toxic response to, poly (amido amine) (PAMAM) dendrimers.

110 These nanoscale aminated dendrimers have a systematically variable molecular structure, and the 
111 homologous series of increasing generation, and concomitant size and number of surface amino

112 groups, has been demonstrated to be ideal to study the dependence of nanoparticle cellular

113 interactions on the physico-chemical properties of the particles. Previous studies have examined

114 the structurally dependent toxicity, and underlying mechanisms of endocytosis, oxidative stress,

115 immune responses and consequent toxicity ${ }^{7,8,9,10}$ and the responses have been numerically

116 modelled, as a guide towards a predictive toxicology approach. ${ }^{10,41}$ For consistency and to allow

117 comparisons with the previous work, the in vitro studies reported here were also carried out

118 using the immortalised human keratinocyte cell line, HaCaT, and identical oxidative stress and

119 cytotoxic assays. Furthermore, PAMAM dendrimers of Generation 4 and 6 were chosen as the

120 extremes of the previously reported cytotoxicological response. In comparison, it is

121 demonstrated that treatment with BSO results in a significant change in the nanoparticle uptake 122 mechanisms and cytotoxicity. 


\section{Materials and Methods}

$124 \underline{\text { Materials }}$

125 DMEM F12 HAM growth medium, Penicillin, Streptomycin, fluorescently labelled, Polystyrene 126 nanoparticles, with amine surface modification $\left(\mathrm{PSNP}_{\mathrm{NH}}\right) 100 \mathrm{~nm}, \mathrm{DL}-\mathrm{Buthionine}-(S, R)$ 127 sulfoximine(BSO) and 3-(4,5-dimethylthiazol-2-yl)-2,5-diphenyltetrazolium bromide (MTT) dye 128 were purchased from Sigma-Aldrich, Ireland. The PAMAM dendrimer nanoparticles, 129 generations 4 (molecular weight: 14,214 g/mol) and 6 (molecular weight: 58,046 g/mol), were 130 purchased from Sigma-Aldrich and manufactured by Dendritech Inc. ThiolTrackerTM Violet

131 (TTV), CellLight ${ }^{\circledR}$ Early Endosomes-RFP, BacMam 2.0, Fetal Bovine Serum (FBS), L-

132 Glutamine, Alamar Blue (AB) and 5-(and-6)-carboxy-2',7'-dichlorodihydrofluorescein diacetate 133 (carboxy- $\mathrm{H}_{2}$ DCFDA) dye were purchased from Life Technologies ${ }^{\mathrm{TM}}$, Bio-Sciences, Ireland.

134 HaCaT cells were purchased from Cell Line Services (CLS), Eppelheim, Germany. TrueLine 96135 well cell culture plates were used for all viability and ROS studies.

136 All fluorescence and absorbance readings were taken on a Molecular Devices SpectraMax M3 137 Spectrometer. Confocal Laser Scanning Fluorescence Microscopy (CLSM) images were taken 138 on a Zeiss LSM 510 Confocal Laser Scanning Microscope and processed using ImageJ software 139 (with co-localisation analysis performed with the JaCoP plugin for ImageJ). All viability, ROS 140 and GSH data analysis was performed using SigmaPlot v10.0 software.

$142 \underline{\text { Methods }}$

$143 \quad$ Cell culture

144 HaCaT cells are an immortalised, non cancerous human keratinocyte cell line and were used for 
145 these experiments. The cells were cultured in DMEM F12 HAM supplemented with 10\% FBS, $14645 \mathrm{IU} / \mathrm{mL}$ penicillin, $45 \mathrm{IU} / \mathrm{mL}$ streptomycin and $2 \mathrm{mM} \mathrm{L}$-glutamine at $37^{\circ} \mathrm{C}$ in $5 \% \mathrm{CO}_{2}$.

147 All assays carried out in this set of experiments were performed in 96 well plates, with cells 148 plated at a concentration of $1 \times 10^{4}$ cells/well in $100 \mu \mathrm{L}$ of DMEM medium. Cells were allowed 24

149 hours to attach and were then treated with BSO for an additional 18 hours (at a concentration of

$150200 \mu \mathrm{M}$ ), after which cells were exposed to PAMAM G4 or G6 dendrimers (in DMEM F12

151 HAM, with 5\%FBS, $45 \mathrm{IU} / \mathrm{mL}$ penicillin, $45 \mathrm{IU} / \mathrm{mL}$ streptomycin, $2 \mathrm{mM}$ L-glutamine and

$152200 \mu \mathrm{M}$ BSO) at various concentrations for the specified time points. Six replicates of each

153 concentration were performed per plate and each plate was repeated in triplicate.

155 ThiolTracker ${ }^{\mathrm{TM}}$ Violet (TTV)

156 ThiolTracker ${ }^{\mathrm{TM}}$ Violet (TTV) is a GSH detection agent. Cells were plated as described above and 157 a concentration gradient of BSO was applied. Cells were left for 18 hours at $37^{\circ} \mathrm{C}$ in $5 \% \mathrm{CO}_{2}$ to 158 allow for reduction of the amount of intracellular GSH. Cells were then washed twice with PBS, $159100 \mu \mathrm{L}$ of TTV dye (at a final concentration of $20 \mu \mathrm{M}$ ) were added to each well and the plates 160 were allowed to incubate at $37^{\circ} \mathrm{C}$ in $5 \% \mathrm{CO}_{2}$ for 30 minutes, after which the TTV solution was

161 removed and replaced with PBS. The fluorescence of each well was then read using the

162 SpectraMax M3 spectrometer with $\lambda_{\mathrm{EX}}=404 \mathrm{~nm}$ and $\lambda_{\mathrm{EM}}=526 \mathrm{~nm}$. GSH values were calculated 163 as compared to the unexposed control.

$165 \quad$ Viability assays

166 Alamar Blue and MTT assays were used to determine the changes in cell viability, after 167 treatment with BSO as described above, as a result of exposure to both PAMAM G4 and G6 
168 dendrimers. Both Alamar Blue and MTT were performed on the same plate. The PAMAM G4 169 concentrations used were: $0.16,0.32,0.65,1.3,2.6,5.2,7.8$ and $10.4 \mu \mathrm{M}$, while the PAMAM G6 170 concentrations were: $0.08,0.16,0.32,0.65,1.3,2.6,3.9$ and $5.2 \mu \mathrm{M}$. The lower initial value of

171 the PAMAM G6 dendrimers was used due to their reported $\mathrm{EC}_{50}$ value being much lower than

172 their G4 counterparts. ${ }^{7,8,9}$ Dose dependent viability percentages were calculated at time points: 6,

$17312,24,48$ and 72 hours. Percentage viability was calculated as compared to a control which had 174 been exposed to $200 \mu \mathrm{M}$ BSO, but had no nanoparticle treatment; this was to ensure any changes

175 were caused by the nanoparticle and were not the result of BSO treatment. A separate control

176 where no BSO was present was also performed and showed there was little difference between

177 cells with no BSO exposure and cells which were exposed to BSO (Supplementary Material, 178 Figure: S1).

$180 \quad$ Alamar Blue (AB)

181 The Alamar Blue assay was made up from 10X stock solution in medium (DMEM F12 HAM, 182 with no additional supplements). At the specified time point, the plates were removed from the 183 incubator and the medium containing PAMAM dendrimer was removed, the cells were washed 184 with $100 \mu \mathrm{L}$ PBS and then $100 \mu \mathrm{L}$ of DMEM F12 HAM(unsupplemented) containing Alamar 185 Blue were added to each well. The plates were incubated for 3 hours at $37^{\circ} \mathrm{C}$ in $5 \% \mathrm{CO}_{2}$ to allow 186 for conversion of the dye. The fluorescence of each well was then read using the SpectraMax M3 187 spectrometer with $\lambda_{\mathrm{EX}}=555 \mathrm{~nm}$ and $\lambda_{\mathrm{EM}}=585 \mathrm{~nm}$. 188

$189 \quad$ MTT (3-(4,5-dimethylthiazol-2-yl)-2,5-diphenyltetrazolium bromide) 
190 A stock solution of MTT was made at a concentration of $0.5 \mathrm{mg} / \mathrm{mL}$. $500 \mu \mathrm{L}$ of this stock were 191 added for every 10mL of medium (DMEM F12 HAM, with no additional supplements). At the

192 specified time point, the plates were removed from the incubator and the medium containing

193 PAMAM dendrimer was discarded, the cells were washed with $100 \mu \mathrm{L}$ PBS and then $100 \mu \mathrm{L}$ of

194 DMEM (unsupplemented) containing MTT was added to each well. The plates were incubated

195 for 3 hours at $37^{\circ} \mathrm{C}$ in $5 \% \mathrm{CO}_{2}$ to allow for conversion of the dye. After 3 hours, any remaining

196 dye was removed and each well was again washed with $100 \mu \mathrm{L}$ PBS, after which $100 \mu \mathrm{L}$ of

197 DMSO was added and the plates were placed on a shaker for 10 minutes to allow for the dye to

198 solubilise. The absorbance of each well was then read using the SpectraMax M3 spectrometer

199 with $\lambda_{\mathrm{ABS}}=595 \mathrm{~nm}$.

200

$201 \quad \underline{\text { Reactive Oxygen Species (ROS) }}$

202 5-(and-6)-carboxy-2',7'-dichlorodihydrofluorescein diacetate (carboxy- $\mathrm{H}_{2} \mathrm{DCFDA}$ ) dye was used

203 for the detection of ROS. The dye was made up to a final concentration of $10 \mu \mathrm{M}$ in sterile PBS.

204 Before addition of PAMAM dendrimer, this dye was added to the cells and allowed to incubate

205 for 1 hour, after which, the dye was removed, the cells were washed thrice with PBS and the

206 medium containing PAMAM dendrimer was added. At the specified time points the fluorescence

207 was read by the SpectraMax M3 spectrometer with $\lambda_{\mathrm{EX}}=488 \mathrm{~nm}$ and $\lambda_{\mathrm{EM}}=535 \mathrm{~nm}$.

208

209 Confocal Laser Scanning Microscopy (CLSM)

210 Cells were plated onto MatTek 35mm glass bottom dishes at a concentration of 20,000 cells/dish

211 in DMEM F12 HAM (supplemented with 10\% FBS, 45 IU/mL penicillin, $45 \mathrm{IU} / \mathrm{mL}$

212 streptomycin and 2mM L-glutamine) and allowed to attach for 4 hours, at which point the 
213 medium was removed and replaced with medium containing Celllight ${ }^{\circledR}$ Early Endosomes-RFP,

214 BacMam 2.0 at a concentration of 20 particles per cell. Early endosome formation was tracked

215 with the Celllight ${ }^{\circledR}$ Early Endosome - RFP kit, which transfects, into the cell, a version of Rab5a

216 with a bound Red Fluorescent Protein. The cells were allowed to incubate $\left(37^{\circ} \mathrm{C}, 5 \% \mathrm{CO}_{2}\right)$ for 16

217 hours to ensure transfection with the early endosome reagent. After this, the medium was

218 removed and cells were washed twice with PBS. For cells being tested without BSO, medium

219 was added for 18 hours (these samples are referred to as untreated cells/untreated controls in the

220 text), while for cells being tested with BSO, medium containing $200 \mu \mathrm{M}$ BSO was added for 18

221 hours. Cells were again washed with PBS and carboxy- $\mathrm{H}_{2}$ DCFDA was added for 1 hour $(10 \mu \mathrm{M}$

222 in 2mL PBS), after which cells were again washed twice with PBS. PAMAM dendrimers were

223 added at a concentration of $3.21 \mu \mathrm{M}(\mathrm{G} 4)$ and $1 \mu \mathrm{M}(\mathrm{G} 6)$ and cells were allowed to incubate for 3

224 hours (G4) or 1 hour (G6) and were then washed twice with PBS and imaged with the Zeiss

225 LSM 510 Confocal Laser Scanning Microscope. 100nm PSNP-NH ${ }_{2}$ with attached Green

226 Florescent Protein was used as a positive control to ensure the Cellight ${ }^{\circledR}$ Early Endosomes-RFP

227 was functioning as expected; the results of this test are available in the supplementary material

228 section, Figure S2. Negative controls were also performed with cells which were not exposed to

229 any nanoparticles, and little to no fluorescence was noted (data not shown). For ROS monitoring,

230 doses and time points noted above were chosen based on the maximum responses previously

231 reported in literature. ${ }^{8}$ All confocal images were analysed using ImageJ and co-localisation

232 studies were performed using Manders split coefficients calculated with the JaCoP plugin for

233 ImageJ. ${ }^{42}$

234

235 Data Analysis and Statistics 
236 Data analysis was performed using SigmaPlot ${ }^{\mathrm{TM}}$ v10.0 and fluorescence was calculated based on

237 the values of BSO controls (which were unexposed to nanoparticles, but had been treated with

$238200 \mu \mathrm{M}$ BSO for 18 hours).

239 "The cytotoxicity, GSH and ROS experiments were performed in 96-well microplates with six

240 replicates per plate and each plate repeated three times. Therefore, data points shown represent

241 the mean of 18 points, with error bars representing \pm the standard deviation (as calculated by

242 SigmaPlot ${ }^{\mathrm{TM}} \mathrm{v} 10.0$ ). Confocal Images were taken on a Zeiss LSM 510 and processed using

243 ImageJ software. Images were taken of eight cells/groups of cells and the images presented in the

244 manuscript are representative of the sampled cell population." 


\section{$246 \underline{\text { Results }}$}

\section{$247 \quad$ BSO treatment}

248 While maximising the desired effect of the BSO on the cells, it is important that the viability of

249 the cells is not affected. TTV showed a 40\% reduction in intracellular levels of GSH for the

$250 \mathrm{HaCaT}$ cells upon $200 \mu \mathrm{M}$ BSO exposure for 18 hours (adapted from the methods of: He et al.

251 (2003)). ${ }^{24}$ This dose and time point were found to have a minimal impact on cellular viability as

252 measured by the AB and MTT assays (data available in Supplementary Material, Figure: S3).

253 Higher concentrations were found to have an effect on cellular viability, although a more

254 pronounced effect was observed by CLSM, where signs of cellular stress were noted (data not

255 shown). Therefore $200 \mu \mathrm{M}$ BSO exposure was chosen as optimal.

\section{$257 \quad$ PAMAM G4 dendrimers}

258 CLSM was employed as a way to examine the effect of BSO treatment on the cellular uptake 259 mechanisms and subsequent oxidative stress. Early endosome formation was tracked with the

260 Celllight ${ }^{\circledR}$ Early Endosome - RFP kit and the formation of ROS was also tracked using the 261 carboxy- $\mathrm{H}_{2}$ DCFDA dye. Fluorescently labelled PSNP- $\mathrm{NH}_{2}$ of $100 \mathrm{~nm}$ diameter were employed 262 as positive controls, and the results for those are shown in the Supplementary Material: Figure 263 S2. 


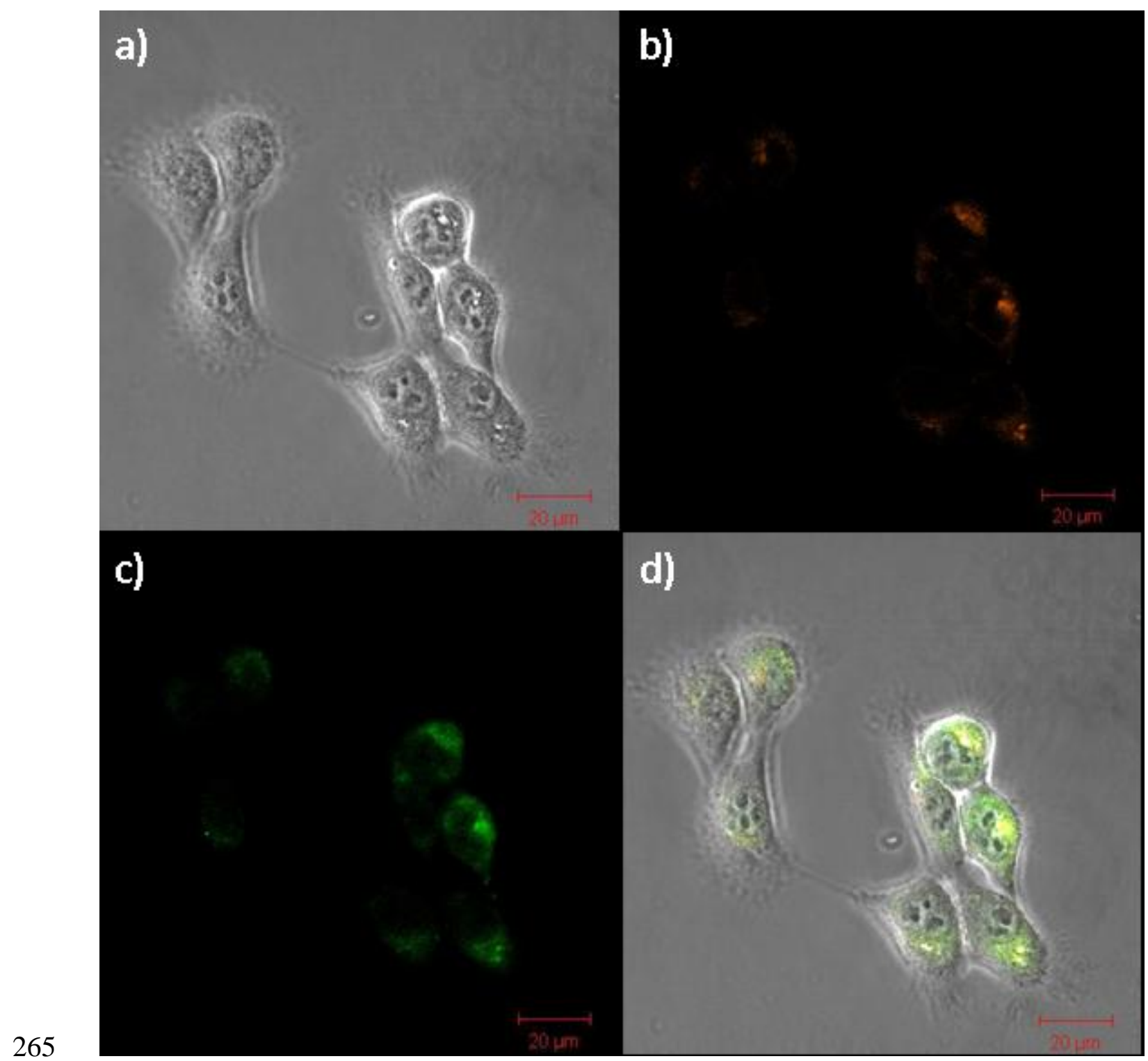

266 Figure 1: CLSM images of HaCaT (live) cells, upon exposure to 3.21 $\mu$ M PAMAM G4

267 dendrimer at 3 hours (no BSO is present in this sample). Image a) shows the bright field image

268 of the cells, Image b) shows the fluorescence generated by the early endosomal red fluorescent

269 protein, Image c) shows the fluorescence generated by the ROS (interacting with the carboxy-

$270 \underline{\mathrm{H}}_{2} \underline{D C F D A}$ dye) and Image d) shows the overlay of images a-c, where yellow coloured areas

271 indicate simultaneous endosomal and ROS activity. 
273 Figure 1 shows the HaCaT cells following the reported paradigm of PAMAM G4 endocytosis

274 and subsequent ROS generation at the endosomal sites. ${ }^{6,7,8,9}$ Co-localisation (performed on the

275 images in Figure 1) shows that $91( \pm 3) \%$ of the generated ROS occurs in the neighbourhood of

276 endosomes, and that $71( \pm 4) \%$ of endosomal formation resulted in increases in ROS production

277 (the other $30 \%$ of endosomal activity is most likely due to endocytosis which would be routinely

278 done by the cell and would not involve the dendrimers and therefore not produce ROS).

279

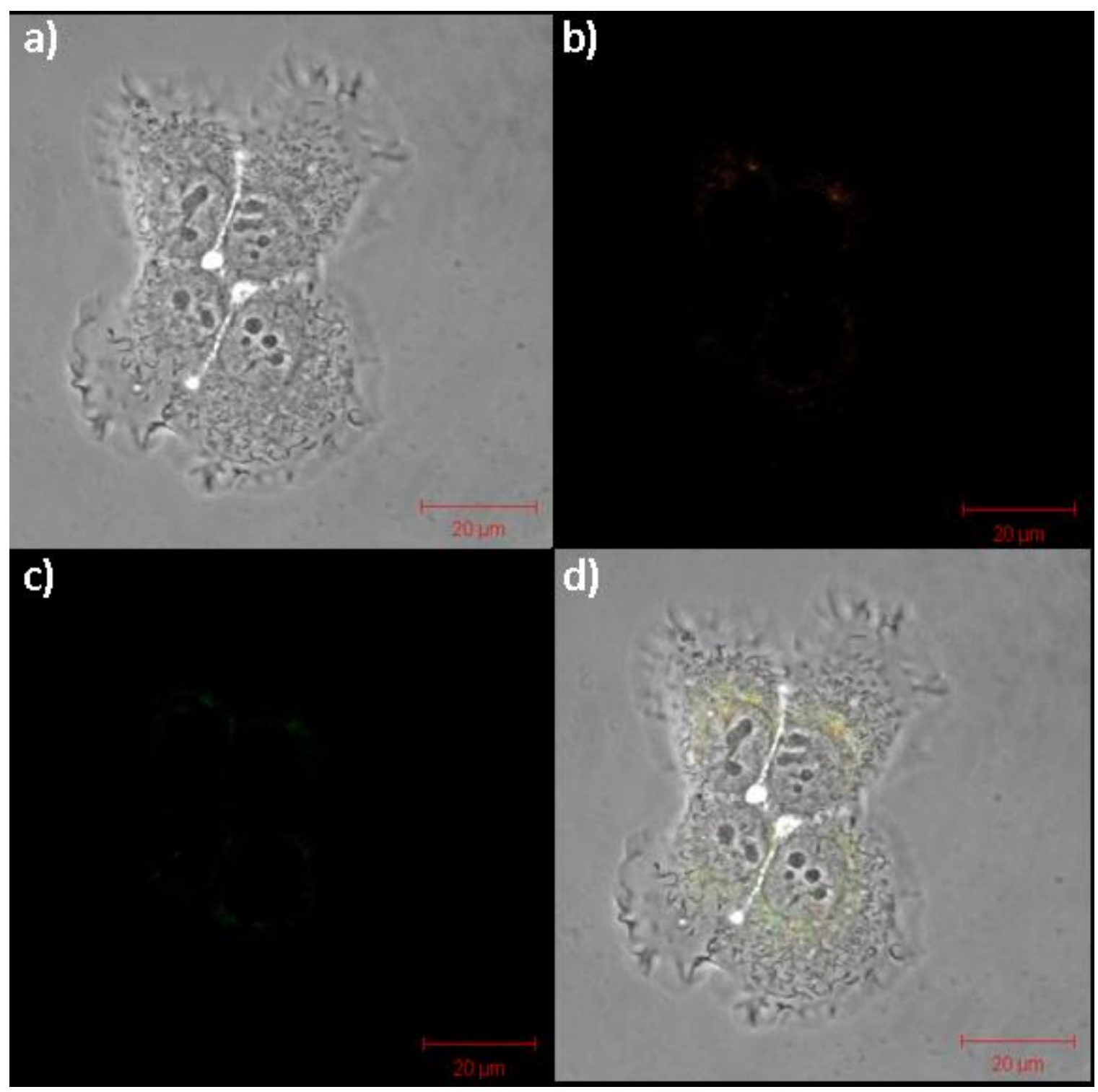


281 Figure 2: Confocal images of HaCaT (live) cells, upon exposure to $3.21 \mu \mathrm{M}$ PAMAM G4

282 dendrimer at 3 hours, with pre-treatment of BSO: $200 \mu \mathrm{M}$ for 18 hours. Image a) shows the bright

283 field image of the cells, Image b) shows the fluorescence generated by the early endosomal red

284 fluorescent protein, Image c) shows the fluorescence generated by the ROS (interacting with the

$285 \underline{\text { carboxy- } \mathrm{H}_{2}} \underline{\underline{D C F D A} \text { dye) and Image d) shows the overlay of images a-c, where yellow coloured }}$

286 areas indicate simultaneous endosomal and ROS activity.

288 In contrast, Figure 2 shows $\mathrm{HaCaT}$ cells which have been treated with $\mathrm{BSO}$ for 18 hours prior to

289 PAMAM G4 exposure (BSO was still present in the media upon exposure, to ensure conditions

290 were consistent). In images (b) and (c), the intensity of the endosomal RFP and ROS dye have

291 been significantly reduced. Again co-localisation analysis was performed (on images in Figure 2)

292 and showed that $30( \pm 6) \%$ of the generated ROS was happening around the endosomes, and that

$29346( \pm 14) \%$ of endosomal formation resulted in increases in ROS production. Intensity analysis of

294 the red fluorescent protein produced by endocytosis, on average, showed a reduction in intensity

295 of $70( \pm 3) \%$ for cells treated with BSO.

296

297 Endosomal uptake was clearly reduced, although a decrease in the intensity of response of the

298 ROS detection dye, carboxy- $\mathrm{H}_{2}$ DCFDA, was also noted, prompting a quantitative analysis of the 299 ROS contents of the HaCaT cells: Figure 3. 


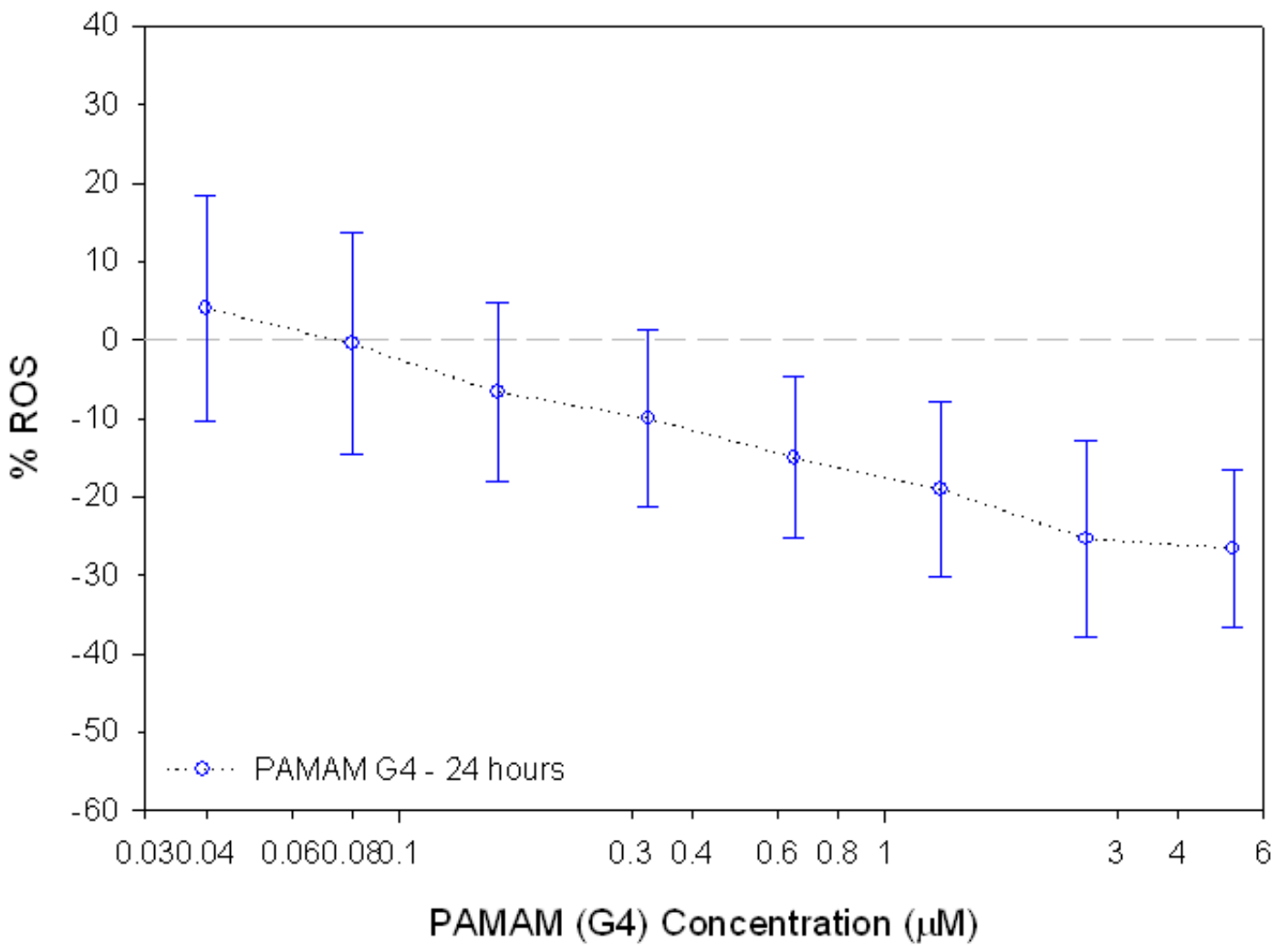

Figure 3: Dose dependant generation of ROS in HaCaT cells (shown for 24 hours for the

303 PAMAM G4). ROS is compared to BSO control which was set to equal 0\%. The X-axis is

304 plotted logarithmically to allow for better visualisation of lower concentrations. Data points are

305 the mean of 18 samples, with error bars showing $( \pm)$ the standard deviation.

307 The analysis of ROS showed a net decrease at all doses tested; for clarity, only the dose

308 dependence of the 24 hour test has been shown, the full data-set is available in the supplementary

309 material section (Figure S4). Over the dose range tested, the ROS response followed a

310 monotonically decreasing trend. This result, is again, in contrast with previous observations, in

311 which ROS levels were seen to increase above control levels in a dose dependant fashion, upon

312 G4 dendrimer exposure. ${ }^{8}$ 
314 To examine how these changes in both endocytosis and ROS production affected the viability of

315 the cell AB and MTT assays were performed. HaCaT cells were exposed to $200 \mu \mathrm{M}$ BSO for 18

316 hours and subsequently exposed to varying concentrations of PAMAM G4 nanoparticles.

317 Viability was measured at 6, 12, 24, 48 and 72 hours (Figure 4 (a) and (b)).

(a)

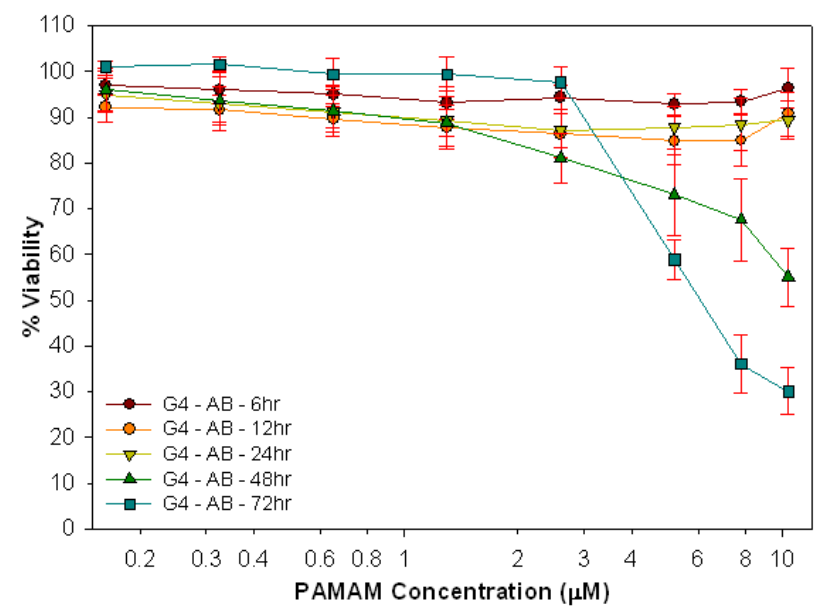

(b)

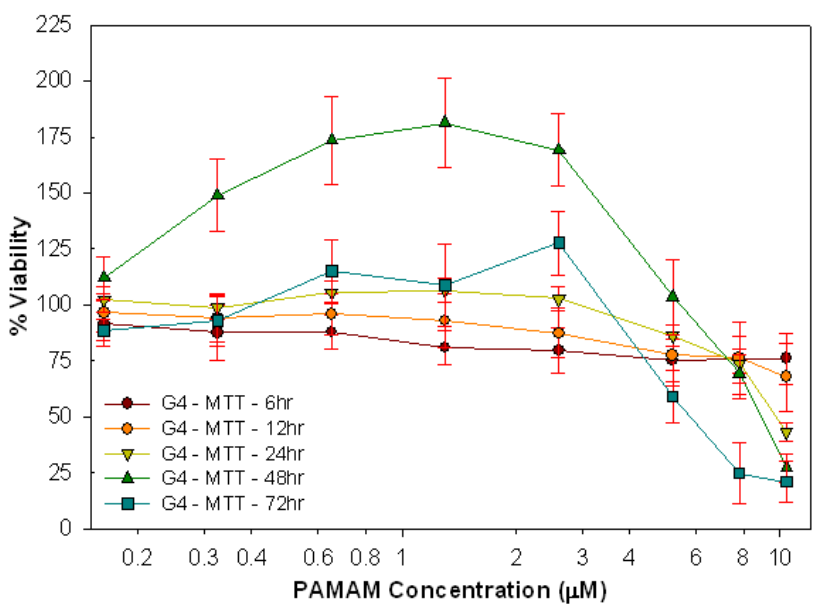

321 Figure 4: Alamar Blue (a) and MTT (b) dose dependant viability results for PAMAM G4

322 dendrimers in HaCaT cells after 6, 12, 24, 48 and 72 hours. Viability is calculated as the

323 percentage of living cells as compared to BSO control. Data points are the mean of 18 samples,

324 with error bars showing $( \pm)$ the standard deviation.

326 The AB assay shows little or no toxicity of PAMAM G4 at time points 6, 12, and 24 hours,

327 whereas the viability is reduced to $50 \%$ at 48 hours and $30 \%$ at 72 hours, for the higher dose

328 exposures. In $\mathrm{HaCaT}$ cells which have not been treated with $\mathrm{BSO}$, the $\mathrm{EC}_{50}$ obtained from $\mathrm{AB}$ is

329 around $10 \mu \mathrm{M}$ at 24 hours, ${ }^{7}$ whereas, in Figure 4, at the same time point, it is clear that there is no

330 significant toxicity. The MTT results show a dramatically different dose dependent cytotoxicity

331 profile for the BSO dosed cells compared to un-dosed. ${ }^{7}$ At 6 hours exposure (Figure 4b), the 
332 viability is seen to be reduced to around $80 \%$ over the entire dose range. However, at exposure

333 times of 12, 24, 48 and 72 hours, increases in viability are seen for the low-medium dose range.

334 The effect is particularly pronounced at the 48 hour time point, at which the recorded MTT

335 response is $\sim 175 \%$ of control, for an exposure dose of $\sim 1 \mu \mathrm{M}$. For doses of $\sim 2.6 \mu \mathrm{M}$ and higher,

336 the MTT assay response registers a decrease in viability, which is more pronounced in the longer

337 time point exposures.

339 PAMAM G6 dendrimers

340 Analysis of uptake and ROS production was again carried out using CLSM (with the same

341 method as the PAMAM G4) incorporating the carboxy- $\mathrm{H}_{2}$ DCFDA dye and Cellight ${ }^{\circledR}$ Early

342 Endosome - RFP kit (Figure 5 and 6). 


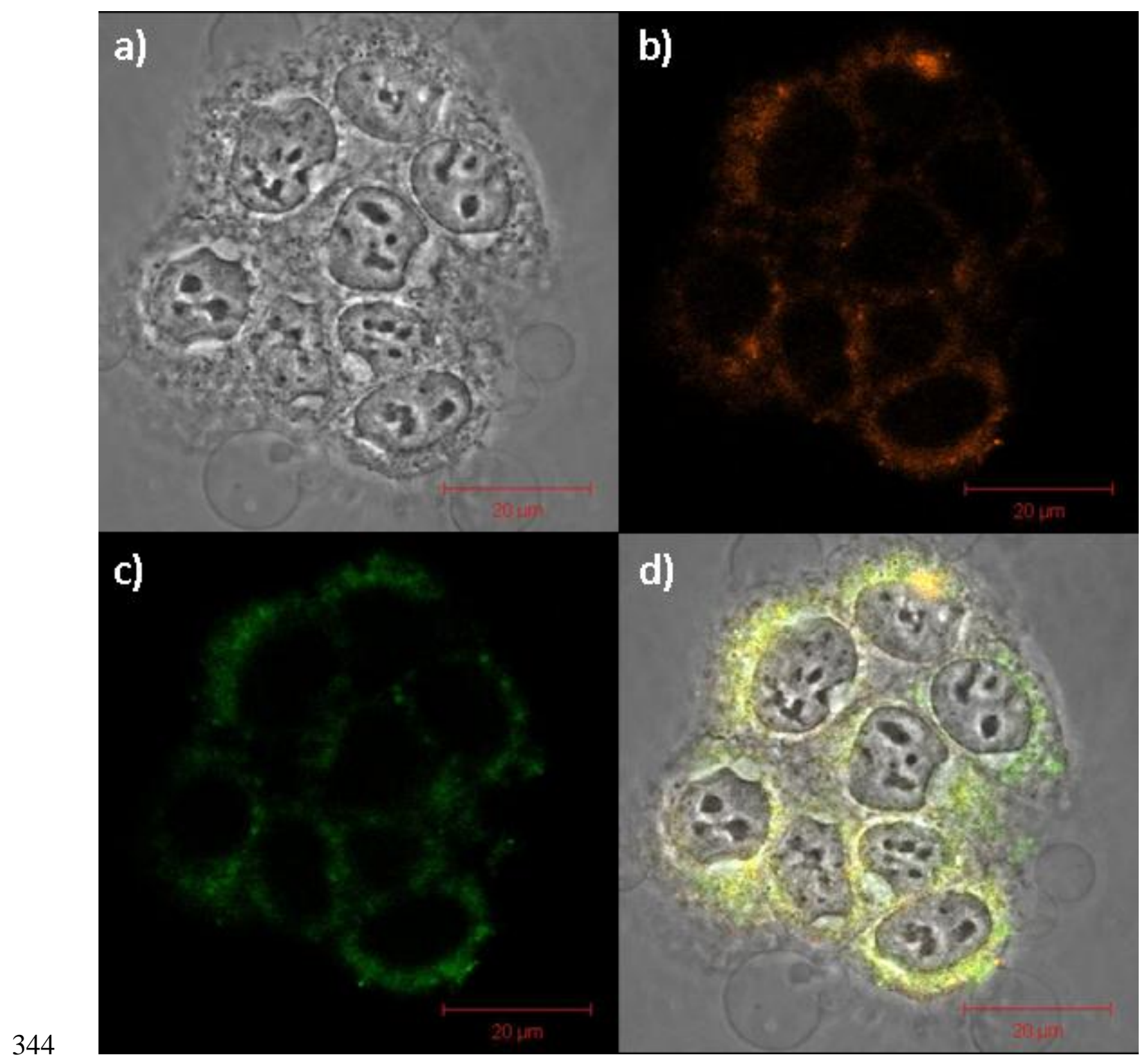

345 Figure 5: Confocal images of HaCaT (live) cells, upon exposure to 1 $\mu \mathrm{M}$ PAMAM G6 dendrimer

346 at 1 hour (no BSO is present in this sample). Image a) shows the bright field image of the cells,

347 Image b) shows the fluorescence generated by the early endosomal red fluorescent protein,

348 Image c) shows the fluorescence generated by the ROS (interacting with the carboxy- ${ }_{2} \underline{\text { DCFDA }}$

349 dye) and Image d) shows the overlay of images a-c, where yellow coloured areas indicate

350 simultaneous endosomal and ROS activity. 
352 In the absence of BSO, PAMAM G6 exposure, resulted in strong red fluorescence indicating a

353 high level of endocytosis, complemented by strong green fluorescence indicating increased ROS

354 production. Co-localisation analysis (of images in Figure 5) found that $75( \pm 2) \%$ of the generated

355 ROS occurred in the region of the endosomes, and that $92( \pm 1) \%$ of endocytosis resulted in

356 increases in ROS production. This strong level of co-localisation is indicative of the accepted

357 paradigm of nanoparticle uptake by endocytosis, and ROS production at the site of endosomes.

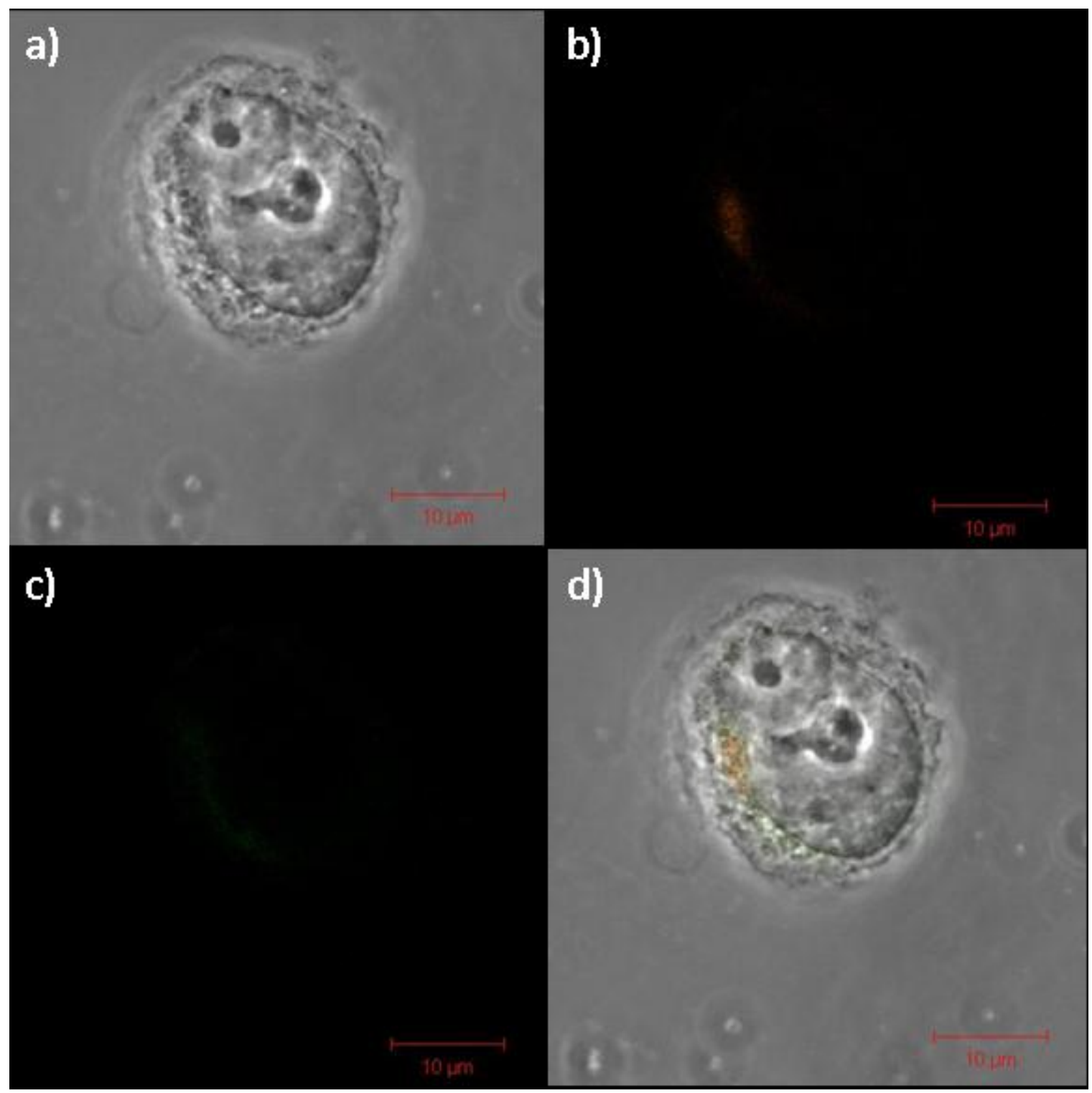


360 Figure 6: Confocal images of $\mathrm{HaCaT}$ (live) cells, upon exposure to $1 \mu \mathrm{M}$ PAMAM G6 dendrimer

361 at 1 hour, with pre-treatment of BSO: $200 \mu \mathrm{M}$. Image a) shows the bright field image of the cells,

362 Image b) shows the fluorescence generated by the early endosomal red fluorescent protein,

363 Image c) shows the fluorescence generated by the ROS (interacting with the carboxy- $\mathrm{H}_{2} \underline{D C F D A}$

364 dye) and Image d) shows the overlay of images a-c, where yellow coloured areas indicate

365 simultaneous endosomal and ROS activity.

367 In comparison to exposure of PAMAM G6 in cells without BSO treatment (untreated cells),

368 there is a sparsity of endosomal activity and oxidative stress response in cells which had been

369 pre-treated with BSO for 18 hours. Analysis (of the images in Figure 6) showed decreased levels

370 of co-localisation between the ROS and endosomal formation: only $41( \pm 18) \%$ of ROS

371 production was recorded in areas where endosomes had formed and $55( \pm 10) \%$ of endocytosis

372 resulted in increases in ROS production. Intensity analysis of the red fluorescent protein

373 produced by endocytosis, on average, showed a reduction in intensity of $61( \pm 1) \%$ for cells

374 treated with BSO when compared to the untreated cells.

375

376 Again, in light of the reduced ROS response, quantitative analysis, using carboxy- $\mathrm{H}_{2} \mathrm{DCFDA}$

377 was performed: Figure 7. 


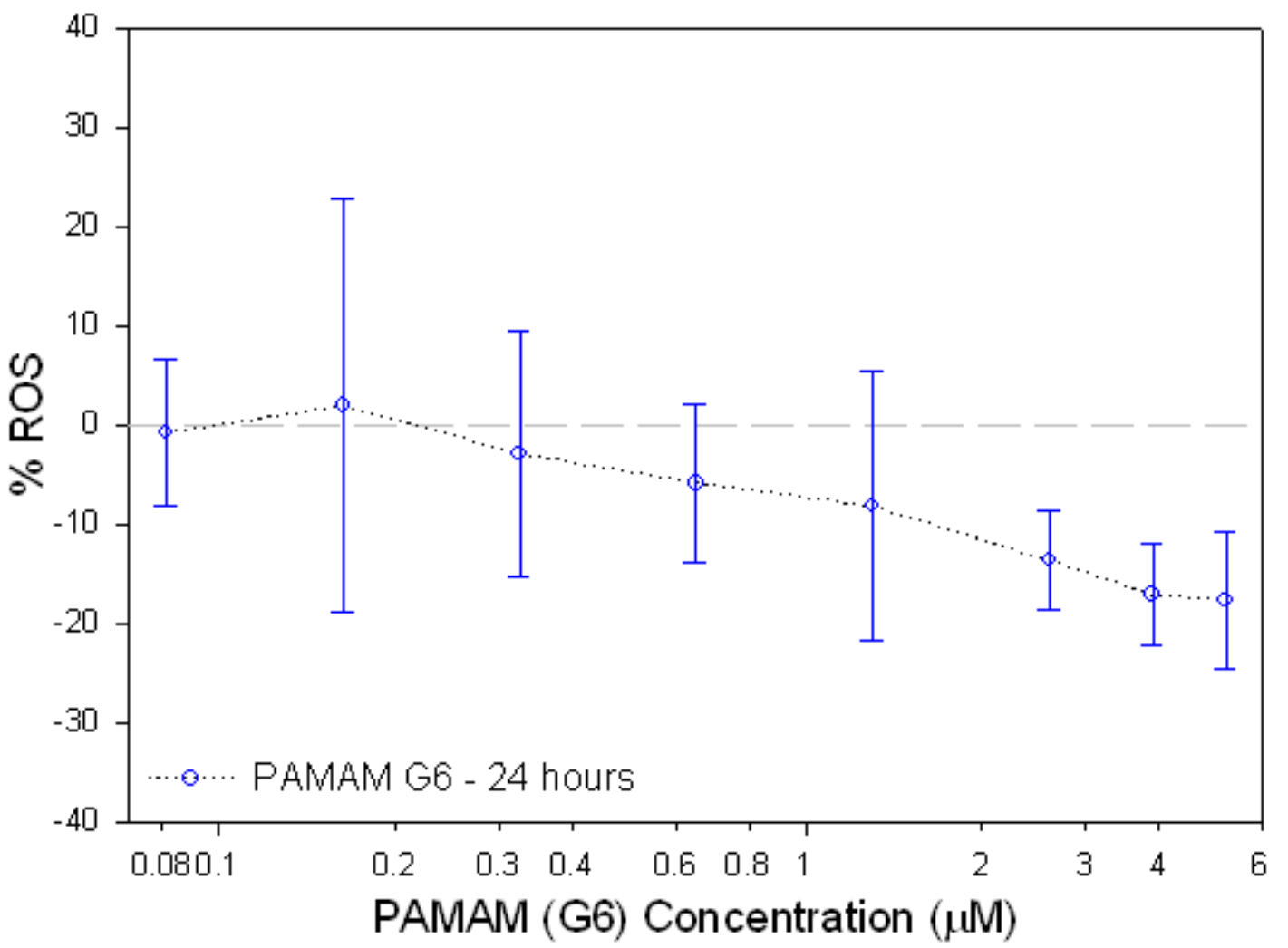

379 Figure 7: Dose dependant generation of ROS in HaCaT cells (shown at 24 hours for the

380 PAMAM G6). ROS is compared to BSO control which was set to equal 0\%. The X-axis is

381 plotted logarithmically to allow for better visualisation of lower concentrations. Data points are

382 the mean of 18 samples, with error bars showing $( \pm)$ the standard deviation.

384 The dose response of the ROS generation shows similarities with the G4 in the overall

385 progression of the response, pointing to a trend where increasing concentration leads to

386 decreasing amounts of ROS, contrary to what has been observed in previous studies without

387 BSO exposure. ${ }^{8}$ As with the G4 analysis, for clarity, only one of the eight concentrations tested

388 have been shown, the full data-set is available in the supplementary material section (Figure S5).

389 At all doses tested, the ROS response followed the same trend as shown in Figure 7. 
391 To analyse the effect this had on viability, AB and MTT assays were performed: Figure 8.
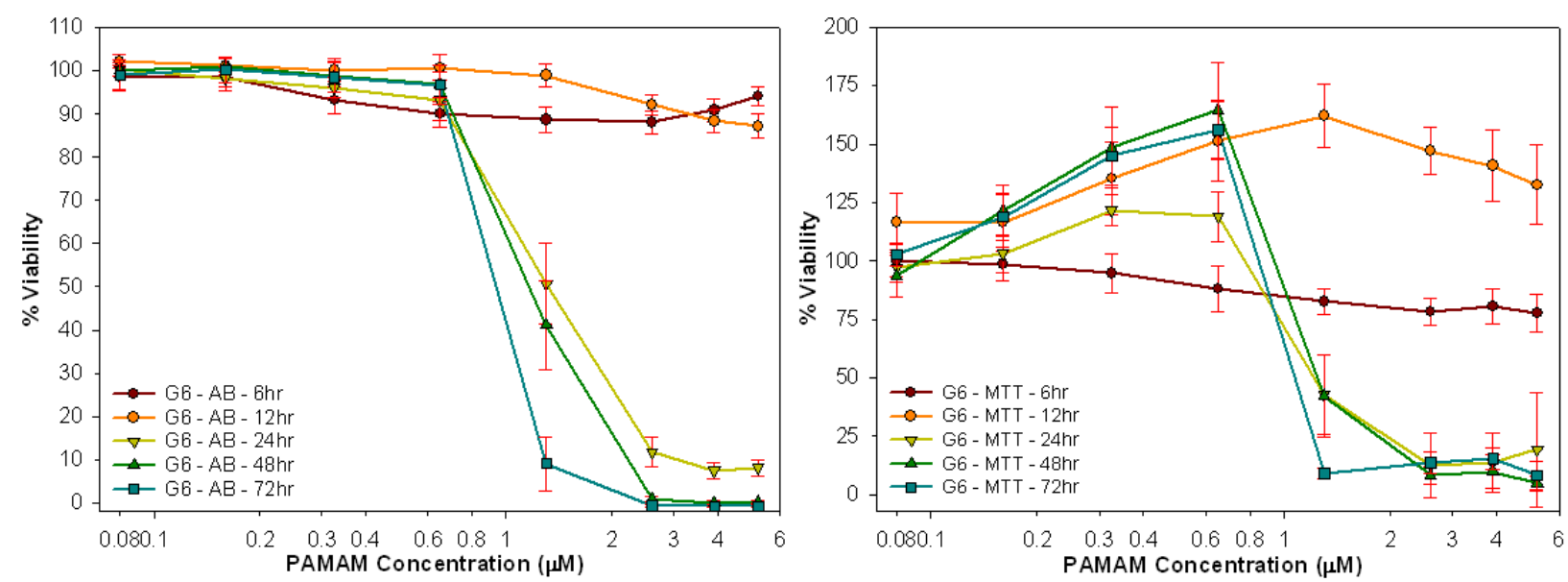

395 Figure 8: Alamar Blue (a) and MTT (b) dose dependant toxicity results for PAMAM G6

396 dendrimers in HaCaT cells after 6, 12, 24, 48 and 72 hours. Viability is calculated as the

397 percentage of living cells as compared to a BSO control. The X-axis is plotted logarithmically to

398 allow for better visualisation of lower doses. Data points are the mean of 18 samples, with error

399 bars showing $( \pm)$ the standard deviation.

401 The viability results of the PAMAM G6 dendrimers are similar to those observed in the G4 402 analysis. In Figure 8, for 6 and 12 hour exposure, little toxicity is recorded by $A B$, although the 403 response is significantly stronger at the later time points. The previously reported $\mathrm{EC}_{50}$ obtained 404 form the AB assay for PAMAM G6 dendrimers is in the range of $1 \mu \mathrm{M}-1.6 \mu \mathrm{M}$ at 24 hours, ${ }^{8}$ 405 which would appear to agree with the values obtained in this study. However, as was the case for 406 PAMAM G4 exposure, the MTT assay registers increased percentage viability, compared to 407 BSO control, for the intermediate doses, for all but the 6 hour exposure time point. A cytotoxic 
408 response is elicited for doses greater than $1 \mu \mathrm{M}$, for the 24,48 and 72 hour time points and the

409 response is consistent with the previously reported $\mathrm{EC}_{50}$ values of $0.92 \mu \mathrm{M}-1.13 \mu \mathrm{M} .^{7}$

$411 \quad$ PAMAM G4-G6 Comparative Analysis

412 PAMAM nanoparticle toxicity is a generation dependant process, ${ }^{7,8,9}$ therefore, a comparison of

413 the response of BSO treated cells to G4 and G6 dendrimer exposure was performed, to establish

414 whether a similar generation dependence of the cellular responses is still evident.

415

416 The AB and MTT assays were compared for PAMAM G4 and G6 dendrimers, and the results

417 are shown in Figures 9 (AB) and 10 (MTT). For both dendrimers, approximately equivalent

418 concentration ranges were used, although, for the PAMAM G6 dendrimers, a slightly lower

419 initial concentration was used due to the higher associated toxicity., ${ }^{7,8,9,10}$

a)

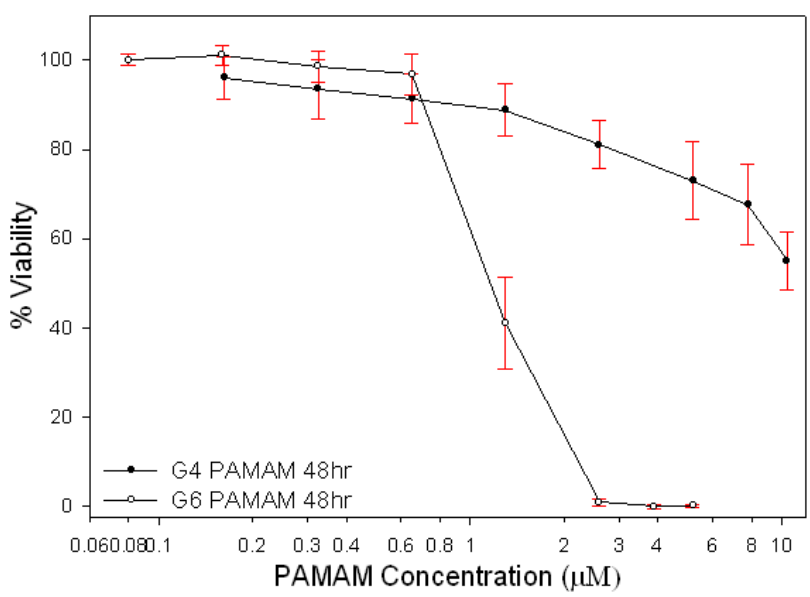

b)

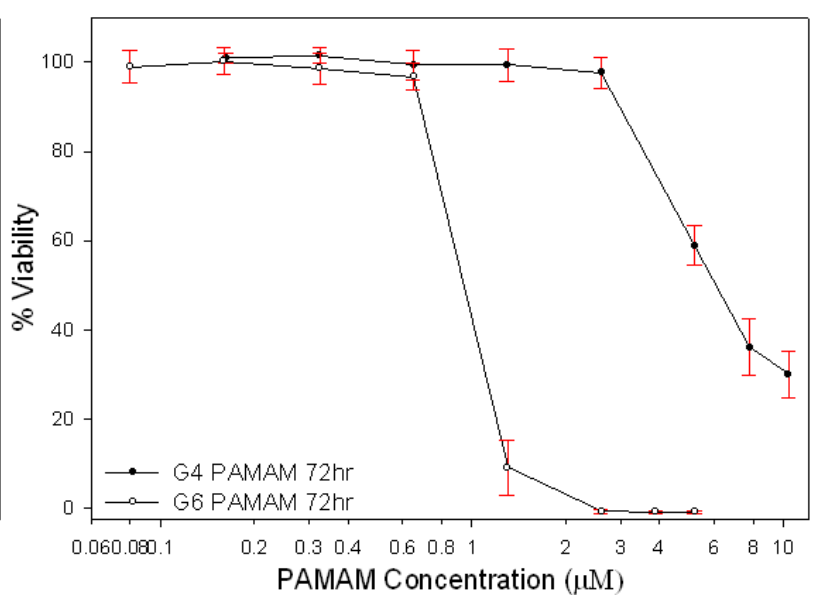

Figure 9: Alamar Blue (AB) dose dependant toxicity results comparing the PAMAM G4 and G6

424 dendrimers in HaCaT cells after 48 hours (a) and 72 hours (b). Viability is calculated as the

425 percentage of living cells as compared to BSO control. The x-axis is plotted logarithmically to 
426 allow for better visualisation of lower doses. Data points are the mean of 18 samples, with error

427 bars showing $( \pm)$ the standard deviation. 6, 12 and 24 hour graphs can be seen in the

428 supplementary material section (Figure S6).

429

430 Little or no significant cytotoxicity was registered by the AB assay for either dendrimer at the 6

431 and 12 hour time points. At 24 hours, the higher toxicity associated with the G6 dendrimers

432 becomes apparent, while the G4 still shows no significant change (Supplementary Material:

433 Figure S6, c). At 48 hours (Figure 9(a)) the G4 dendrimers begin to elicit a significant toxic

434 response and finally, at 72 hours (Figure 9(b)), the G4 toxic profile is beginning to match that of

435 G6, although the reduction of viability is much higher for G6.

436

437 a)

b)
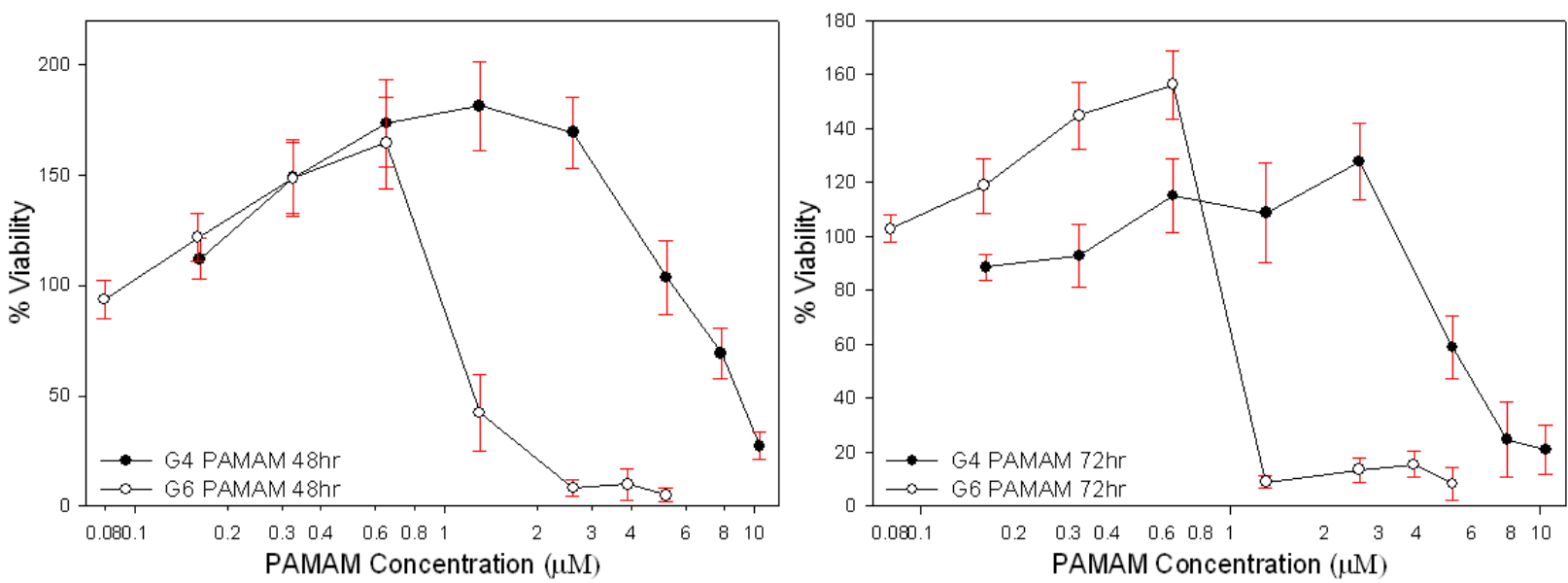

Figure 10: MTT dose dependant toxicity results comparing the PAMAM G4 and G6 dendrimers

440 in HaCaT cells after 48 hours (a) and 72 hours (b). Viability is calculated as the percentage of

441 living cells as compared to BSO control. The X-axis is plotted logarithmically to allow for better

442 visualisation of lower doses. Data points are the mean of 18 samples, with error bars showing $( \pm)$ 
443 the standard deviation. 6,12 and 24 hour graphs can be seen in the supplementary material

444 section (Figure S7).

445

446 In the MTT, similar to AB response, no significant difference was seen in the 6 hour analysis for

447 the two dendrimers, although differences become apparent at 12 hours (Supplementary Material:

448 Figure S7, b), at which point the MTT response increases above that of controls for the G6

449 dendrimer. This increase in MTT response also occurs for the G4 dendrimers, although, it is not

450 manifest until the later time point of 48 hours (Figure 10(a)). Similar to the AB response,

451 cytotoxicity is registered by the MTT assay for both dendrimers beginning at 24 hours; however,

452 the $\mathrm{AB}$ only reaches about $50 \%$ viability. At 48 and 72 hours a more complete toxic profile for

453 both assays is seen and distinct generation dependence is observable, more consistent with that

454 observed for untreated cells. ${ }^{7}$

455 


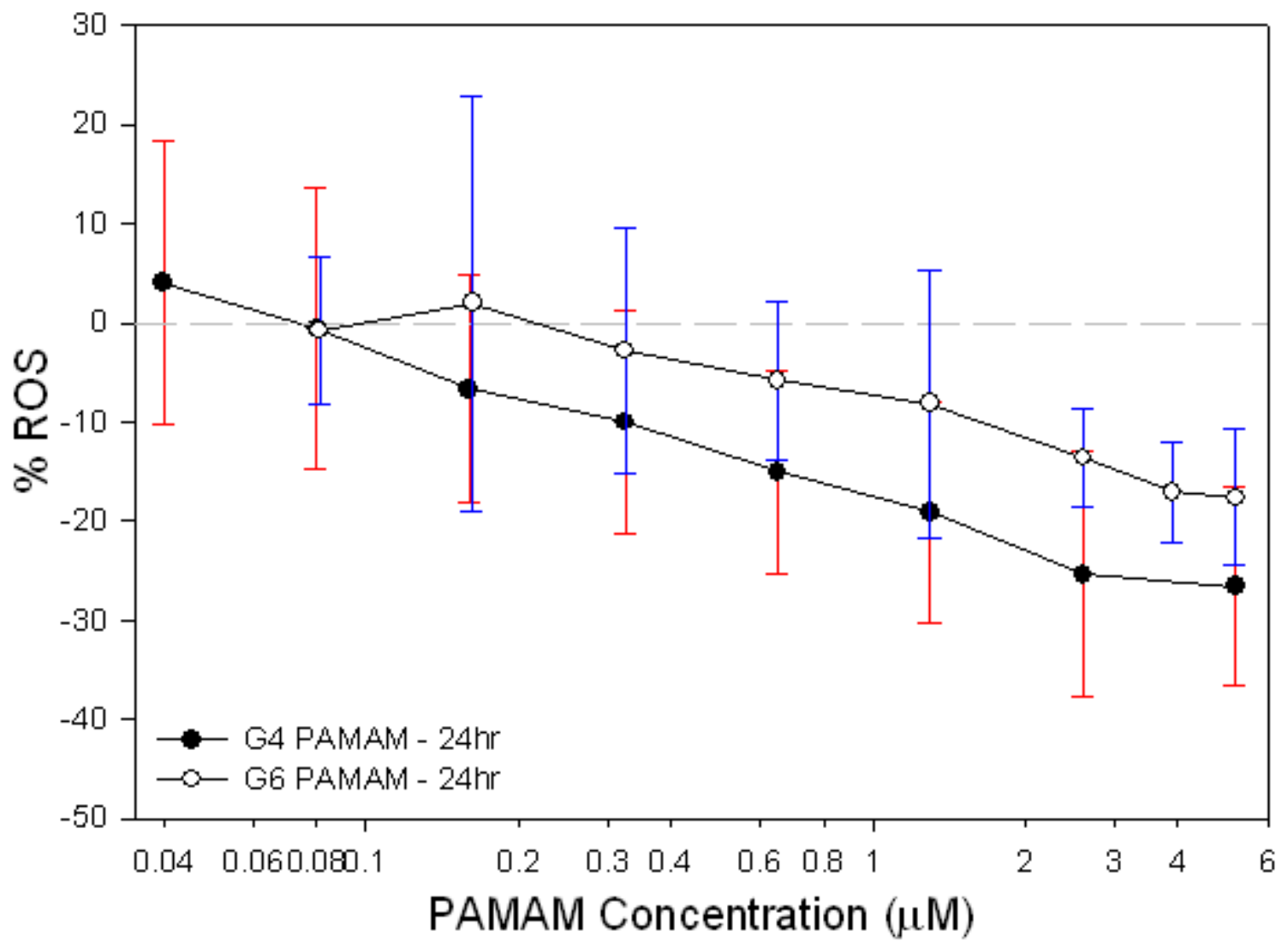

457 Figure 11: Dose dependant generation of ROS in HaCaT cells (shown at 24 hours for PAMAM

458 G4 and G6). Data is compared to BSO control which was set to equal 0\%. The X-axis is plotted

459 logarithmically to allow for better visualisation of lower concentrations. Data points are the mean

460 of 18 samples, with error bars showing $( \pm)$ the standard deviation.

462 To further investigate any generation dependence, the dose response of the ROS was compared 463 for the G4 and the G6 (Figure 11). It is notable that the generation dependence of the trends is 464 reversed in Figure11 where G4 elicits a more pronounced reduction in ROS levels than G6. 


\section{$\underline{\text { Discussion }}$}

467 The confocal images of Figures: 1 and 2 (PAMAM G4) and Figures: 5 and 6 (PAMAM G6)

468 show that, for cells treated with $200 \mu \mathrm{M}$ BSO for 18 hours prior to PAMAM exposure, the rate of

469 endocytosis has been markedly reduced. This reduction in endocytosis is accompanied by a

470 similar reduction in intracellular ROS and a dramatic change in the responses of the cytotoxic

471 assays. Nevertheless, the observed reduction of intracellular ROS and cytotoxic responses are

472 systematically dependent on dendrimer exposure time, dose and generation, consistent with the

473 intracellular action of the dendritic nanoparticles.

475 The demonstrated mechanism of PAMAM dendrimer toxicity is one of endocytosis, ${ }^{8,43,44}$ ROS

476 production, ${ }^{7,8,9,18}$ subsequent endosomolysis, whereby the nanoparticle bursts out of the

477 endosome/lysosome into the cytosol, ${ }^{19}$ and localisation in the mitochondria. ${ }^{45}$ Mukherjee and

478 Byrne (2013) identified two apoptotic pathways, the death-receptor pathway (extrinsic, Fas

479 mediated FADD pathway ${ }^{46}$ ) and the mitochondrial pathway (intrinsic, TNF- $\alpha$ mediated

$\left.480 \mathrm{FADD}^{47}\right){ }^{10}$ The former is initiated by the earlier stage ROS generation in the region of the

481 endosomes, while the latter is initiated by the localisation of the dendrimers in the mitochondria.

482 It has been proposed that the early stage ROS production is due to the action of NADPH

483 oxidase $^{6}$ (producing superoxide anions $\left.\left(\mathrm{O}_{2}^{-}\right)^{48}\right)$ and the v-ATPase proton pump (providing

484 protons, ${ }^{49}$ ultimately leading to production of $\mathrm{H}_{2} \mathrm{O}_{2}{ }^{50}$ in and around the endosome. In cells

485 treated with BSO, however, PAMAM dendrimers elicit a dramatically different cytotoxicity

486 profile, as registered by the AB and MTT assays, compared to that of untreated cells. ${ }^{7,8}$ 
488 Studies by Khalid et al. (2015) of cellular uptake of PPI dendrimers have demonstrated that,

489 although the larger generation PPI dendrimers are endocytosed and elicit similar responses to

490 PAMAM equivalents in $\mathrm{HaCaT}$ cells, for smaller generation PPI dendrimers, uptake by passive

491 diffusion occurs and, when the dendrimers enter the cell in this way, they were observed to act as

492 antioxidants and elicit a significantly reduced cytotoxic effect. ${ }^{18}$ BSO exposure has been shown

493 to permeabilise the cell membrane ${ }^{28}$ and as a result, it is proposed that the PAMAM G4 and G6

494 dendrimers are able to circumvent the endocytotic process, are uptaken by passive diffusion, and,

495 as PAMAM dendrimers are similar in structure to PPI dendrimers and have comparable surface

496 chemistry, similarly behave as antioxidants in the cytosol, eliciting substantially reduced

497 cytotoxic responses.

499 Alamar Blue is a non-specific assay and measures cellular viability based on the overall activity

500 of the cytosolic environment. ${ }^{51}$ The significant reduction, rather than expected increase, of

501 toxicity as registered by this assay reflects the reduction in the endocytosis process (which

502 initiates the Fas mediated FADD (death-receptor) apoptotic pathway), in favour of the passive

503 diffusion of nanoparticles across the membrane, and consequent reduction in ROS generation in

504 the region of endosomes. However, endocytosis is not fully eliminated for either generation, and

505 therefore, the activation of the Fas mediated pathway, on a much reduced scale, would in part

506 explain why the generation dependent response is still observed for the PAMAMs.

508 The MTT assay measures mitochondrial activity as an indicator of cellular viability ${ }^{52,53,54}$ and, in

509 the case of the studies described here, the mitochondria are implicated in at least two different

510 processes and changes in MTT responses reflect the dose and generation dependence of these 
511 processes at several time points. The first process is the loss of GSH from the cell, which has

512 been shown to cause activation of mitochondrial signalling pathways and expression of genes

513 associated with apoptosis, growth and differentiation. ${ }^{33,38,39,40}$ This overall increase in

514 mitochondrial activity (observed in the low dose regime), is seen as the initial increase in MTT

515 values above controls, associated with dose and generation dependent decrease in ROS below

516 controls, due to the antioxidant effect of the passively uptaken dendrimer nanoparticles.

517

518 The second effect gives rise to a sharp decrease in mitochondrial activity (observed in the higher

519 dose regime); consistent with PAMAM dendrimer localisation and disruption of the

520 mitochondria, ${ }^{7,8}$ initiating the mitochondrial apoptotic pathway, leading to cell death in a dose

521 dependant fashion, as observed for untreated cells. ${ }^{7,8}$ This process may be accelerated via the

522 opening of the mitochondrial membrane permeability transition pore. ${ }^{30,31,32}$ Whether passively

523 diffused into the cell, or released into the cytosol by endosomolysis after endocytosis, the result

524 of free PAMAM dendrimers in the cytosol and subsequent localisation to the mitochondria

525 should be equivalent, both resulting in disruption of the mitochondria, a second phase increase in

526 ROS within the cell, subsequent decay in the mitochondrial membrane potential and finally the

527 initiation of a cascade leading to apoptosis. ${ }^{7,8,9,10}$ It should also be noted that in both cases the

528 opening of the mitochondrial membrane transition pore occurs, either by the action of

$529 \mathrm{BSO},{ }^{30,31,32}$ or due to the release of the endosomal/lysosomal contents causing intracellular

530 release of $\mathrm{Ca}^{2+}$, leading to calcium dependant opening of the pore. ${ }^{21}$ This would, in both cases,

531 facilitate the entry of the dendrimer to the mitochondria. As a result, in the high dose regime, the

532 observed toxic response of the BSO treated cells, as registered by the MTT assay, is not very

533 different to that observed for untreated cells. 
535 The passive diffusion of dendrimers across the cell membrane is a size dependant process ${ }^{18}$ and,

536 the generation dependence of the cellular responses to the two PAMAM dendrimer generations,

537 G4 and G6, (Figures: 1, 2, 5 and 6), is consistent with a higher uptake rate for the G4 dendrimer

538 than the G6. The greater reduction of intensity of Rab-5a-RFP compared to controls (with no

539 BSO treatment), G4 dendrimers (70\%) compared to G6 (60\%), indicates a higher diffusion rate

540 for G4 dendrimers, leaving fewer available for endocytosis. This further explains the higher rate

541 of anti-oxidative activity exhibited by the G4 dendrimers when compared to G6 (Figure 11).

542 Overall, it would appear that the membrane has become more permeable to an extent that favours

543 passive uptake, although not completely eliminating active endocytosis.

545 Within the framework of Adverse Outcome Pathways (AOPs), recently endorsed by the

$546 \mathrm{OECD}^{55,56}$ as a method to simplify the representation of the mode of action of a toxicant or

547 agonist, the generation of ROS can be considered the key Molecular Initiating Event (MIE) of

548 the AOP, which ultimately leads to the AO of loss of cell viability. The treatment with BSO

549 causes a depletion of GSH, which would lead to the expectation of much increased ROS levels

550 after endocytosis. However, that was not observed and the result of the reduction of intrinsic

551 GSH levels by BSO treatment was predominantly the increased permeability of the cell

552 membrane, resulting in an increased rate of uptake of the PAMAM dendrimers by passive

553 diffusion, making it a favoured uptake mechanism, more so for the smaller G4 dendrimer than

554 the larger G6. The co-existence of the parallel uptake mechanisms increases the complexity of

555 any model to describe the in vitro system, although it could prove a useful model to develop

556 networks of AOPs, initiated by different MIEs. However, to fully examine the effect of reduction 
557 of GSH in terms of decreased anti-oxidant activity alone, an assay which did not cause decrease

558 of the permeabilisation of the cell membrane would be necessary.

560 Considering the potential for PAMAM dendrimers in nanomedical applications, it is important to 561 note that, when diffused into the cell, the aminated surface chemistry of the dendrimers lend 562 them antioxidant activity, similar to small molecular anti-oxidants, such as $\mathrm{N}$-acetylcysteine 563 (NAC) and NAC amide (NACA - a more bio-available version of NAC). NACA has been

564 extensively studied as an antioxidant in the cell, due to its ability to diffuse across the membrane 565 and the presence of a terminal proton donor group. ${ }^{57,58}$ Interestingly, it has also been shown to 566 completely reverse the damage caused to the cell by depletion of GSH. ${ }^{59,60,61}$ NACA, due to this 567 strong anti-oxidant ability, has been proposed in the treatment of several disorders and diseases, 568 such as: HIV ${ }^{62}$ Alzheimer's and Parkinson's disease, ${ }^{60,63}$ cataract formation, ${ }^{59}$ retinal 569 degeneration $^{64}$ and essentially any disease where ROS is identified as the potential MIE. (See: 61 and 570 references therein) If PAMAM dendrimers are seen to act in a similar way, it may potentially allow for 571 their use in a plethora of different nano-medical applications. As a strategy for drug release, 572 endosomolysis can be extremely disruptive to the cell ${ }^{20}$ and therefore, in the case of cationic 573 nanoparticles for intracellular nanomedical applications, avoiding the process of endocytosis 574 may be a valid strategy to pursue. ${ }^{22}$ In terms of therapeutic applications, direct entry into the 575 cytosol may be a more convenient route for drug or gene delivery. 


\section{Conclusions}

578 Although PAMAM dendritic nanoparticles are known to elicit significant cytotoxic responses in

579 vitro, the cellular response mechanisms can be notably altered by treatment of the cells with

580 BSO. The treatment increases the cell membrane permeability, enabling uptake of the particles

581 by passive diffusion, where after, they act as antioxidants in the cytosol, rather than producing

582 oxidative stress in the region of endosomes. The ability to tune the cellular uptake mechanism

583 allows direct entry into the cytosol and may have important implications for nanotoxicity as well

584 as drug and gene delivery using nanovehicles.

585 


\section{Acknowledgements}

587 The authors would like to thank Dr. Alan Casey and Esen Efeoglu for their contributions in the 588 capturing and processing of the confocal images for this manuscript.

589

$590 \quad$ Funding Sources

591 Irish Government's Programme for Research in Third Level Institutions, Cycle 4, National

592 Development Plan 2007-2013, supported by the European Union Structural Fund. M Maher is

593 funded through the DIT Fiosraigh President's Award for Research Excellence 2010

594

595 Declaration of interest

596 The authors declare no conflict of interest related to the work presented in this manuscript. 


\section{References}

599 1: Eichman, J.D., Bielinska, A.U., Kukowska-Latallo, J.F. and Baker, J.R.Jr. (2000) The use of

600 PAMAM dendrimers in the efficient transfer of genetic material into cells. J. Pharm. Sci.

601 Technol. 3(7): 233-245.

602

603 2: Duncan, R. and Izzo, L. (2005) Dendrimer biocompatibility and toxicity. Adv. Drug. Deliv.

604 Rev. 57(15): 2215-2237.

605

606 3: De Jong, W. H. and Borm, P.J.A. (2008) Drug delivery and nanoparticles: Applications and 607 hazards. Int. J. Nanomedicine. 3(2): 133-149.

608

609 4: Nel, A., Xia, T., Mädler, L. and Li, N. (2006) Toxic potential of materials at the nanolevel.

$610 \quad$ Science. 311(5761): 622-627.

611

612 5: Jain, K.K., Kesharwani, P., Gupta, U. and Jain, N.K. (2010) Dendrimer toxicity: Let's meet

613 the challenge. Int. J. Pharm. 394(1-2): 122-142.

614

615 6: Xia, T., Kovochich, M., Brant, J., Hotze, M., Sempf, J., Oberley, T., Sioutas, C., Yeh, J.I.,

616 Wiesner, M.R. and Nel, A.E. (2006) Comparison of the Abilities of Ambient and Manufactured

617 Nanoparticles to Induce Cellular Toxicity According to an Oxidative Stress Paradigm. Nano Lett. 618 6(8): 1794-1807.

619 
620 7: Mukherjee, S.P., Davoren, M. and Byrne, H.J. (2010) In vitro mammalian cytotoxicological

621 study of PAMAM dendrimers - towards quantitative structure activity relationships. Toxicol. in

622 Vitro, 24(1): 169-177.

623

624 8: Mukherjee, S.P., Lyng, F.M., Garcia, A., Davoren, M. and Byrne H.J. (2010) Mechanistic 625 studies of in vitro cytotoxicity of Poly(amidoamine) dendrimers in mammalian cells. Toxicol.

626 Appl. Pharmacol. 248(3): 259-268.

627

628 9: Naha, P.C., Davoren, M., Lyng, F.M. and Byrne, H.J. (2010) Reactive Oxygen Species (ROS)

629 induced cytokine production and cytotoxicity of PAMAM dendrimers in J774A.1 cells. Toxicol.

630 Appl. Pharmacol. 246(1-2): 91-99

631

632 10: Mukherjee S.P. and Byrne, H.J. (2013) Polyamidoamine Dendrimer Nanoparticle

633 Cytotoxicity, Oxidative Stress, Caspase Activation and Infammatory Response: Experimental

634 Observation and Numerical Simulation. Nanomed. Nanotech. Biol. Med. 9(2): 202-211.

635

636 11: Anguissola, S., Garry, D., Salvati, A., O’Brien, P.J. and Dawson, K.A. (2014) High Content

637 Analysis Provides Mechanistic Insights on the Pathways of Toxicity Induced by Amine-

638 Modified Polystyrene Nanoparticles. PLoS ONE, 9(9): e108025.

639 doi:10.1371/journal.pone.0108025

640

641 12: Paget, V., Dekali, S., Kortulewski, T., Grall, R., Gamez, C., Blazy, K., Aguerre-Chariol, O., 642 Chevillard, S., Braun, A., Rat, P and Lacroix, G. (2015) Specific Uptake and Genotoxicity 
643 Induced by Polystyrene Nanobeads with Distinct Surface Chemistry on Human Lung Epithelial

644 Cells and Macrophages. PLoS ONE, 10(4): e0123297. doi:10.1371/journal.pone.0123297

645

646 13: Lunov, O., Syrovets, T., Loos, C., Beil, J., Delacher, M., Tron, K., Nienhaus, G.U.,

647 Musyanovych, A., Mailänder, V., Landfester, K. and Simmet, T. (2011) Differential Uptake of

648 Functionalized Polystyrene Nanoparticles by Human Macrophages and a Monocytic Cell Line.

649 ACS Nano. 5(3): 1657-1669.

650

651 14: Nabeshi, H., Yoshikawa, T., Matsuyama, K., Nakazato, Y., Tochigi, S., Kondoh, S., Hirai,

652 T., Akase, T.,Nagano, K., Abe, Y., Yoshioka, Y., Kamada, H., Itoh, N., Tsunoda, S. and

653 Tsutsumi, Y. (2011) Amorphous nanosilica induce endocytosis dependent ROS generation and

654 DNA damage in human keratinocytes. Part. Fibre Toxicol. 8(1): 10 pages.

655

656 15: Mozdzan, M., Szemraj, J., Rysz, J., Stolarek, R.A. and Nowak, D. (2006) Anti-oxidant

657 activity of spermine and spermidine re-evaluated with oxidising system involving iron and

658 copper ions. Int. J. Biochem. Cell Biol. 38(1): 69-81.

659

660 16: Lotito, S.B. and Frei, B. (2004) Relevance of apple polyphenols as antioxidants in human

661 plasma: contrasting in vitro and in vivo effects. Free Radic. Biol. Med. 36(2): 201-211.

662

663 17: Hipkiss AR. (2009) Carnosine and its possible roles in nutrition and health. Adv. Food Nutr.

664 Res. 57(-): 87-154.

665 
18: Khalid, H., Mukherjee, S.P., O’Neill, L. and Byrne, H.J. (2015) Structural dependence of the

667 In vitro cytotoxicity, oxidative stress and uptake mechanisms of Poly(propylene imine) dendritic

668 nanoparticles. J. Appl. Toxicol. (in press - Accepted: October 2015)

670 19: Watson, P., Jones, A.T. and Stephens, D.J. (2005) Intracellular trafficking pathways and drug 671 delivery: fluorescence imaging of living and fixed cells. Adv. Drug Deliver. Rev. 57(1): 43-61.

673 20: Mukherjee, S.P. (2012) Towards Structure Activity Relationships for in vitro Toxicity of 674 Polyamidoamine Dendritic Nanoparticles. Thesis (PhD), Dublin Institute of Technology: 2012. 675 Dublin: DIT.

677 21: Xia. T., Kovochich, M., Liong, M., Mädler, L., Gilbert, B., Shi, H., Yeh, J.I., Zink, J.I. and 678 Nel, A.E. (2008) Comparison of the mechanism of toxicity of zinc oxide and cerium oxide 679 nanoparticles based on dissolution and oxidative stress properties. ACS Nano. 2(10): 2121-2134.

681 22: Guarnieri, D., Sabella, S., Muscetti, O., Belli, V., Malvindi, M.A., Fusco, S., De Luca, E., 682 Pompa, P.P. and Netti, P.A. (2014) Transport across the cell-membrane dictates nanoparticle fate 683 and toxicity: a new paradigm in nanotoxicology. Nanoscale. 6(17): 10264-10273.

685 23: Girard, P-M., Graindorge, D., Smirnova, V., Rigolet, P., Francesconi, S., Scanlon, S. and 686 Sage, E. (2013) Oxidative Stress in Mammalian Cells Impinges on the Cysteines Redox State of 687 Human XRCC3 Protein and on Its Cellular Localization. PLoS ONE, DOI:

688 10.1371/journal.pone.0075751 
690 24: He, Y-Y., Huang, J-L., Ramirez, D.C. and Chignell, C.F. (2003) Role of Reduced

691 Glutathione Efflux in Apoptosis of Immortalized Human Keratinocytes Induced by UVA. J.

692 Biol. Chem. 278(10): 8058-8064.

693

694 25: Madesh, M., Benard, O. and Balasubramanian, K.A. (1998) Glutathione modulates lipid 695 composition of human colon derived HT-29 cells. Int. J. Biochem. Cell Biol. 30(12): 1345-1352.

697 26: Tobi, S.E., Paul, N. and McMillan T.J. (2000) Glutathione modulates the level of free 698 radicals produced in UVA-irradiated cells. J. Photochem. Photobiol. B. 57(2-3): 102-112.

700 27: Li, X.Y., Donaldson, K., Rahman, I. and Mac Nee, W. (1994) An investigation of the role of 701 glutathione in increased epithelial permeability induced by cigarette smoke in vivo and in vitro. 702 Am. J. Respir. Crit. Care Med. 149(6): 1518-1525.

703

704 28: Zaman, G.J., Lankelma, J., van Tellingen, O., Beijnen, J., Dekker, H., Paulusma, C., Oude 705 Elferink, R.P., Baas, F. and Borst, P. (1995) Role of glutathione in the export of compounds from 706 cells by the multidrug-resistance-associated protein. Proc. Natl. Acad. Sci. USA. 92(17): 76907077694.

708

709 29: Griffith, O.W. (1982) Mechanism of Action, Metabolism, and Toxicity of Buthionine 710 Sulfoximine and Its Higher Homologs, Potent Inhibitors of Glutathione Synthesis. J. Biol. Chem. $711 \quad 257(22):$ 13704-13712. 
713 30: Armstrong, J.S. and Jones, D.P. (2002) Glutathione depletion enforces the mitochondrial

714 permeability transition and causes cell death in Bcl-2 overexpressing HL60 cells. FASEB J.

715 16(10): 1263-1265.

716

717 31: Chernyak, B.V. and Bernardi, P. (1996) The mitochondrial permeability transition pore is

718 modulated by oxidative agents through both pyridine nucleotides and glutathione at two separate 719 sites. Eur. J. Biochem. 238(3): 623-630.

720

721 32: Reed, D.J. and Savage, M.K. (1995) Influence of metabolic inhibitors on mitochondrial 722 permeability transition and glutathione status. Biochim. Biophys. Acta. 1271(1): 43-50.

724 33: Circu, M.L. and Aw, T.Y. (2008) Glutathione and apoptosis. Free Radic. Res. 42(8): 689725706.

726

727 34: Lash, L.H. (2006) Mitochondrial Glutathione Transport: Physiological, Pathological and 728 Toxicological Implications. Chem. Biol. Interact. 163(1-2): 54-67.

730 35: Beatrice, M.C., Stiers, D.L. and Pfeiffer, D.R. (1984) The role of glutathione in the retention 731 of $\mathrm{Ca}^{2+}$ by liver mitochondria. J. Biol. Chem. 259(2): 1279-1287. 
733 36: Lotscher, H.R., Winterhalter, K.H., Carafoli, E. and Richter, C. (1979) Hydroperoxides can

734 modulate the redox state of pyridine nucleotides and the calcium balance in rat liver

735 mitochondria. Proc. Natl. Acad. Sci. USA. 76(9): 4340-4344.

736

737 37: Olafsdottir, K., Pascoe, G.A. and Reed, D.J. (1988) Mitochondrial glutathione status during 738 Ca2+ ionophore-induced injury to isolated hepatocytes. Arch. Biochem. Biophys. 263(1): 226739235.

741 38: Davis, W.Jr., Ronai, Z. and Tew, K.D. (2001) Cellular thiols and reactive oxygen species in 742 drug-induced apoptosis. J. Pharmacol. Exp. Ther. 296(1): 1-6.

744 39: Petit, P.X., Susin, S.A., Zamzami, N., Mignotte, B. and Kroemer, G. (1996) Mitochondria 745 and programmed cell death: Back to the future. FEBS Lett. 396(1): 7-13.

747 40: Skulachev, V.P. (1996) Why are mitochondria involved in apoptosis? Permeability transition

748 pores and apoptosis as selective mechanisms to eliminate superoxide-producing mitochondria 749 and cells. FEBS Lett. 397(1): 7-10.

750

751 41: Maher, M.A., Naha, P.C., Mukerjee, S.P. and Byrne, H.J. (2014) Numerical simulations of in 752 vitro nanoparticle toxicity - the case of Poly(amido amine) dendrimers. Toxicol. In Vitro. 28(8): $753 \quad 1449-1460$. 
755 42: Zinchuk, V. and Zinchuk, O. (2008) Quantitative colocalization analysis of confocal

756 fluorescence microscopy images. Curr. Protoc. Cell Biol. Ch.4(Unit 4.19), 1-16.

758 43: Kitchens, K.M., Foraker, A.B., Kolhatkar, R.B., Swaan, P.W., Ghandehari, H. (2007)

759 Endocytosis and interaction of poly (Amidoamine) dendrimers with Caco-2 cells. Pharmaceut.

760 Res. 24(11): 2138-2145.

761

762 44: Hong, S., Bielinska, A.U., Mecke, A., Keszler, B., Beals, J.L., Shi, X., Balogh, L., Orr, B.G.,

763 Baker, J.R.Jr and Banaszak Holl, M.M. (2004) Interaction of poly(amidoamine) dendrimers with

764 supported lipid bilayers and cells: hole formation and the relation to transport. Bioconjugate

765 Chem. 15(4): 774-782.

766

767 45: Lee, J.H., Cha, K.E., Kim, M.S., Hong, H.W., Chung, D.J., Ryu, G. and Myung, H. (2009)

768 Nanosized polyamidoamine (PAMAM) dendrimer-induced apoptosis mediated by mitochondrial 769 dysfunction. Toxicol. Lett. 190(2): 202-207.

770

771 46: Wajant, H. (2002) The Fas signaling pathway: more than a paradigm. Science

772 296(5573): 1635-1636.

773

774 47: Desagher, S. and Martinou, J.C. (2000) Mitochondria as the central control point of 775 apoptosis. Trends Cell Biol. 10(9): 369-377.

776 
777 48: Shuvaev, V.V., Han, J., Yu, K.J., Huang, S., Hawkins, B.J., Madesh, M., Nakada, M. and

778 Muzykantov, V.R. (2011) PECAM-targeted delivery of SOD inhibits endothelial inflammatory 779 response. FASEB J. 25(1): 348-357.

780

781 49: Nel, A.E., Mädler, L., Velegol, D., Xia, T., Hoek, E.M.V., Somasundaran, P., Klaessig, F., 782 Castranova, V. and Thompson, M. (2009) Understanding biophysicochemical interactions at the 783 nano-bio interface. Nature Materials. 8(7): 543 - 557.

784

785 50: Babior, B.M., Lambeth, J.D. and Nauseef, W. (2002) The neutrophil NADPH oxidase. Arch. 786 Biochem. Biophys. 397(2): 342-344.

787

788 51: O'Brien, J., Wilson, I., Orton, T. and Pognan, F. (2000) Investigation of the Alamar Blue 789 (resazurin) fluorescent dye for the assessment of mammalian cell cytotoxicity. Eur. J. Biochem. $790 \quad 267(17): 5421-5426$.

791

792 52: Mosmann, T. (1983) Rapid colorimetric assay for cellular growth and survival: Application 793 to proliferation and cytotoxicity assays. J. Immunol. Methods. 65(1): 55-63.

794

795 53: Denizot, F. and Lang, R. (1986) Rapid colorimetric assay for cell growth and survival:

796 Modifications to the tetrazolium dye procedure giving improved sensitivity and reliability. $J$.

797 Immunol. Methods. 89(2): 271-277.

798 
799 54: Hansen, M.B., Nielsen, S.E. and Berg, K. (1989) Re-examination and further development of 800 a precise and rapid dye method for measuring cell growth/cell kill. J. Immunol. Methods. 119(2):

$801 \quad 203-210$.

802

803 55: Organisation for Economic Co-Operation and Development (OECD): Guidance Document 804 on the Validation of (Quantative) Structure Activity Relationships [(Q)SAR] Models, OECD, $805 \quad E N V / J M / M O N O(2007) 2,2007$.

806

807 56: Organisation for Economic Co-Operation and Development (OECD): Guidance Document 808 On Developing and Assessing Adverse Outcome Pathways, OECD, ENV/JM/MONO(2013)6, 8092013.

810

811 57: Price, T.O., Uras, F., Banks, W.A. and Ercal, N. (2006) A novel antioxidant N-acetylcysteine 812 amide prevents gp120- and Tat-induced oxidative stress in brain endothelial cells. Exp. Neurol. 813 201(1): 193-202.

814

815 58: Grinberg, L., Fibach, E., Amer, J. and Atlas, D. (2005) N-acetylcysteine amide, a novel cell 816 permeating thiol, restores cellular glutathione and protects human red blood cells from oxidative 817 stress. Free Radic. Biol. Med. 38(1): 136-145.

818

819 59: Carey, J.W., Pinarci, E.Y., Penugonda, S., Karacal, H. and Ercal, N. (2011) In vivo inhibition 820 of l-buthionine-(S,R)-sulfoximine-induced cataracts by a novel antioxidant, $\mathrm{N}$-acetylcysteine 821 amide. Free Radic. Biol. Med. 50(6): 722-729. 
823 60: Penugonda, S., Mare, S., Goldstein, G., Banks, W.A. and Ercal, N. (2005) Effects of N-

824 acetylcysteine amide (NACA), a novel thiol antioxidant against glutamate-induced cytotoxicity

825 in neuronal cell line PC12. Brain Research. 1056(2): 132-138.

826

827 61: Sunitha, K., Hemshekhar, M., Thushara, R.M., Santhosh, M.S., Yariswamy, M., Kemparaju,

828 K. and Girish, K.S. (2013) N-Acetylcysteine amide: a derivative to fulfill the promises of N-

829 Acetylcysteine. Free Radic. Res. 47(5): 357-367.

830

831 62: Banerjee, A., Zhang, X., Manda, K.R., Banks, W.A. and Ercal, N. (2010) HIV proteins

832 (gp120 and Tat) and methamphetamine in oxidative stress-induced damage in the brain: potential

833 role of the thiol antioxidant N-acetylcysteine amide. Free Radic. Biol. Med. 48(10): 1388-1398.

835 63: Lee, K.S., Kim, S.R., Park, H.S., Park, S.J., Min, K.H., Lee, K.Y., Choe, Y.H., Hong, S.H.,

836 Han, H.J., Lee, Y.R., Kim, J.S., Atlas, D. and Lee, Y.C. (2007) A novel thiol compound, N-

837 acetylcysteine amide, attenuates allergic airway disease by regulating activation of NF-kappaB

838 and hypoxia-inducible factor-1alpha. Exp. Mol. Med. 39(6): 756-768.

839

840 64: Schimel, A.M., Abraham, L., Cox, D., Sene, A., Kraus, C., Dace, D.S., Ercal, N. and Apte,

841 R.S. (2011) N-acetylcysteine amide (NACA) prevents retinal degeneration by up-regulating

842 reduced glutathione production and reversing lipid peroxidation. Am. J. Pathol. 178(5): 2032-

8432043. 